\title{
Scalable and modular wireless-network infrastructure for large-scale behavioural neuroscience
}

\author{
Raza Qazi ${ }^{1,2,13}$, Kyle E. Parker $\mathbb{1}^{3,4,5,6,13}$, Choong Yeon Kim ${ }^{1,13}$, Ruediger Rill7, Makenzie R. Norris $3,4,5,6,8$, \\ Jaeyoon Chung7, John Bilbily3,4,5,6,9, Jenny R. Kim ${ }^{3,4,5,6}$, Marie C. Walicki ${ }^{3,4,5,6}$, Graydon B. Gereau ${ }^{3,4,5,6}$, \\ Hyoyoung Lim7, Yanyu Xiong ${ }^{10}$, Jenna R. Lee ${ }^{11}$, Melissa A. Tapia' ${ }^{12}$, Alexxai V. Kravitz $\mathbb{1}^{9}$, \\ Matthew J. Will'2, Sangtae Ha ${ }^{7 凶}$, Jordan G. McCall $\mathbb{B}^{3,4,5,6,8 凶}$ and Jae-Woong Jeong ${ }^{1 凶}$
}

\begin{abstract}
The use of rodents to acquire understanding of the function of neural circuits and of the physiological, genetic and developmental underpinnings of behaviour has been constrained by limitations in the scalability, automation and high-throughput operation of implanted wireless neural devices. Here we report scalable and modular hardware and software infrastructure for setting up and operating remotely programmable miniaturized wireless networks leveraging Bluetooth Low Energy for the study of the long-term behaviour of large groups of rodents. The integrated system allows for automated, scheduled and real-time experimentation via the simultaneous and independent use of multiple neural devices and equipment within and across laboratories. By measuring the locomotion, feeding, arousal and social behaviours of groups of mice or rats, we show that the system allows for bidirectional data transfer from readily available hardware, and that it can be used with programmable pharmacological or optogenetic stimulation. Scalable and modular wireless-network infrastructure should facilitate the remote operation of fully automated large-scale and long-term closed-loop experiments for the study of neural circuits and animal behaviour.
\end{abstract}

$\mathrm{B}$ ehavioural and circuit neuroscience is labour intensive and often requires researchers to be physically present to conduct experiments in limited numbers of animals at once. This presence inherently alters the observed system. This 'observer effect' is both widespread and often underappreciated in behavioural neuroscience $^{1-5}$. Furthermore, coordinating experiments with large numbers of experimental subjects with precise control is exceptionally challenging, thus making reproducible and repeatable high-throughput in vivo experiments difficult. Recent advances in device engineering and wireless technology have enabled miniaturized, wireless neural devices to help mitigate issues associated with traditionally tethered schemes that can restrict animals' natural movements ${ }^{6-13}$. However, these wireless approaches have substantial disadvantages that have limited their widespread adoption in biomedical research laboratories. Notably, the wireless control of these devices is commonly not selective for a particular animal or device function. To illustrate, it is as if the user is in an electronics store and all the televisions are the same brand. When a remote is used to change the channel on one television, the channel changes on all of them. In addition, many of the existing wireless technologies are also line-of-sight limited (that is, the television remote must be able to directly see the television), requiring the user to be physically present nearby and making high-throughput experiments impossible. While there are instances when one may want to use the same neural manipulations across animals, this limitation is not ideal for more complex behaviours (such as the control of the neural circuitry of two or more socially interacting rodents or animals when performing independent experiments in the same facility) or large experiments testing multiple conditions (that is, frequency-response or dose-response curves). Furthermore, these systems often rely on expensive and highly specialized equipment that may not be portable to other uses, and the available methods are not easily modifiable or scalable for automated or high-throughput experimentation. Overcoming these latter limitations will be necessary to enable large-scale experiments and increase reproducibility across tests.

Recent engineering efforts have produced several highthroughput neuroscience tools (Supplementary Table 1$)^{14-18}$. However, these tools lack true scalability, versatility and customizability to allow researchers to use them across a wide variety of neuroscience studies. They only allow a very limited and specific application (such as movement sensing ${ }^{17}$, olfactory sensing ${ }^{16}$ or touch sensing ${ }^{18}$ ), offer minimal or no wireless range ${ }^{14,16-18}$ and/or require increased number of bulky hardware setups for larger-scale

'School of Electrical Engineering, Korea Advanced Institute of Science and Technology, Daejeon, Republic of Korea. ${ }^{2}$ Department of Electrical, Computer and Energy Engineering, University of Colorado, Boulder, CO, USA. ${ }^{3}$ Department of Anesthesiology, Washington University in St. Louis, St. Louis, MO, USA. ${ }^{4}$ Department of Pharmaceutical and Administrative Sciences, University of Health Sciences and Pharmacy in St. Louis, St. Louis, MO, USA. ${ }^{5}$ Center for Clinical Pharmacology, University of Health Sciences and Pharmacy in St. Louis and Washington University School of Medicine, St. Louis, MO, USA. ${ }^{6}$ Washington University Pain Center, Washington University in St. Louis, St. Louis, MO, USA. 'Department of Computer Science, University of Colorado Boulder, Boulder, CO, USA. ${ }^{8}$ Division of Biology and Biomedical Sciences, Washington University School of Medicine, St. Louis, MO, USA. ${ }^{9}$ Department of Psychiatry, Washington University in St. Louis, St. Louis, MO, USA. ${ }^{10}$ Department of Electrical and Computer Engineering, University of Illinois at Urbana-Champaign, Urbana, IL, USA. "Interdisciplinary Neuroscience Program, University of Missouri, Columbia, MO, USA. ${ }^{12}$ Department of Psychological Sciences, University of Missouri, Columbia, MO, USA. ${ }^{13}$ These authors contributed equally: Raza Qazi, Kyle E. Parker, Choong Yeon Kim. 凶e-mail: sangtae.ha@colorado.edu; jordangmccall@wustl.edu; jjeong1@kaist.ac.kr 
controls $^{14-18}$. These limitations in existing systems considerably hinder the ability to conduct diverse high-throughput neuroscience experiments.

To overcome these limitations, we introduce a remotely programmable, globally accessible hardware and software infrastructure of miniaturized wireless networks, which we refer to as the 'Wireless Network for Behavioural Neuroscience' (WNBN). This infrastructure enables remote, scalable, modular and chronic high-throughput neuroscience studies. Using a custom-designed user interface and internet server for remote access, Bluetooth mesh technology for simultaneous and selective multicontrol (that is, a single remote for simultaneous and independently targeted control of a spatially distributed aggregate of wireless devices) and affordable consumer hardware to enable mass deployment, the WNBN can be easily integrated into existing laboratory infrastructures at scale. Here we describe the operational concepts of the WNBN ecosystem for large-scale in vivo control of freely behaving animals and provide proof-of-principle demonstrations of this ecosystem in established models of behavioural and circuit neuroscience. These approaches provide both a portable and built-in universal system that streamlines experimental workflows and enables previously untenable multiplexing of neural and behavioural manipulations. The WNBN enables perturbations in home cages and social interaction environments, and automates experimentation through a web-based schedule system.

\section{Results}

Concept and operation principle of WNBN for high-throughput in vivo neuroscience research. Figure 1 illustrates the operational concept of the WNBN ecosystem. The WNBN system integrates wireless neural devices ${ }^{6,7,19,20}$ and other laboratory equipment with Bluetooth Low Energy (BLE) and Internet technologies to enable semi-automated and fully automated behavioural studies, respectively. The WNBN can either be controlled locally through BLE piconets (ad hoc network that connect the primary node and multiple secondary nodes using Bluetooth technology ${ }^{21}$ ) using a smartphone (Fig. 1a), or can be accessed globally over the Internet (Fig. 1b; see Table 1 for detailed features and comparison to existing wireless technologies). Use of BLE for both options enables selective simultaneous control of multiple devices (Supplementary Fig. 1). This integrated approach exploits the unique features of both BLE and Internet protocols, which allow energy efficient control of many different neural devices, behavioural assays or other relevant lab equipment in the vicinity $(\sim 100 \mathrm{~m})$ and remote access to the large local networks over the Internet for high-throughput control, respectively. More specifically, the BLE piconet is useful for rapid creation of traditionally small experimental cohorts ( 15 devices) with selective device and output controls, particularly when the experimenter is physically present to conduct the experiment. On the other hand, the Internet protocol and webserver on the minicomputer (Raspberry Pi 3 Model B, Raspberry Pi Foundation; Supplementary Fig. 2) allow researchers to control and receive feedback from many more devices in multiple piconet networks both selectively and simultaneously from any remote, Internet-connected part of the world (Fig. 1b-e). Using this scheme, researchers anywhere in the world can securely connect to the WNBN of neural devices, behavioural apparatuses or other lab equipment located inside a minicomputer-equipped lab. Multiple credentialed users with appropriate administrator permissions can $\log$ on to a secure private server using a browser on a PC, tablet or smartphone to set up desired experiments. Versatile graphical user interfaces (GUIs) of both the smartphone app ${ }^{19}$ and the webserver facilitate this process by enabling manipulation of control parameters for multiple simultaneous experiments (Supplementary Figs. 3 and 4, and Videos 1 and 2). The entire system is economi$\mathrm{cal}$ and portable, only requiring commercial hardware in the form of a smartphone for local control or a minicomputer for global remote access.

This WNBN infrastructure allows the user to remotely set up individual, simultaneous or scheduled sequences of commands (Fig. 1c and Supplementary Video 3). This enables experimental parameters to be sent instantly in real time or scheduled at a specific date and time. These commands are received over the Internet by a BLE-enabled minicomputer. Once the received command is processed and the time of triggering is reached, BLE modules in the minicomputer send this command selectively to the large-scale network of wirelessly connected animals implanted with BLE-enabled neural devices or BLE-enabled traditional equipment. In addition, the WNBN infrastructure supports bidirectional control and sensing of information between the remote user and the devices used in various laboratory experiments (Fig. 1d). This capability can be used to check the current status of environmental parameters (such as temperature, pressure and humidity) before remotely triggering a sensitive behavioural experiment. The data-sending and receiving ability can be further extended to form multiple closed-loop control systems, where the WNBN ecosystem, based on received environmental, behavioural or neural data, will send a command signal to a pre-specified set of devices to perform automated actions (for example, photostimulation, drug delivery, etc.). Lastly, the WNBN ecosystem leverages this unique ability to notably enhance experimental throughput by allowing a remote user to have simultaneous selective control over a variety of experiments within a laboratory, as well as across multiple laboratories (Fig. 1e). In summary, all these functions of the WNBN infrastructure - remote control, selective and simultaneous control, bidirectional communication and device-independent scalable manipulation of both wireless and conventional tethered equipment - are powerful features that can address needs within the neuroscience community for efficient, labour-saving technology and can greatly facilitate high-throughput in vivo neuroscience research.

WNBN architecture and signal flow. Figure 2a,b highlights the network architecture of the WNBN controls, compared to conventional Bluetooth-based schemes. Conventional Bluetooth controls rely on one-to-one node control (Fig. 2a(i),b(i)). In this simple architecture, a single wireless transmitter or controller (that is, remote control centre (RCC)) can wirelessly control a single wireless receiver (that is, remote control module (RCM)). Bluetooth single device control technology provides unique advantages, such as a large wireless control range, no line-of-sight and obstacle handicaps, and low cost and setup time for at-large implementation across laboratories. However, the low-throughput efficiency of this single device control scheme makes it unsuitable for large-scale in vivo neuroscience.

To overcome this limitation, we developed the WNBN technology, including operation modes for both local piconet (Fig. 2a(ii),b(ii)) and global Internet control (Fig. 2a(iii),b(iii)) to enable seamless scalability and ease of implementation across laboratories, as well as devices around the globe. In local piconet WNBN control, we employed a single RCC device to simultaneously control multiple wireless RCMs (up to 15 devices at a time) in its vicinity $(<100 \mathrm{~m})$. In simple terms, it can be visualized as a star network of multiple RCMs forming multiple single device control connections with a single central RCC (Fig. 2a(ii)). Using BLE wireless protocols, we developed the local control system, which synchronizes multiple data streams by the frequency hopping spread spectrum technique, where it multiplexes the serial data flowing from a single RCC to multiple local RCM receivers. Various receivers in the local piconet WNBN are particularly distinguished from one another through the unique 48-bit Bluetooth device address of each RCM, which is available to RCC upon establishment of a local piconet WNBN. We used this identification technique to enable local piconet mode to control devices simultaneously and selectively with $100 \%$ accuracy 


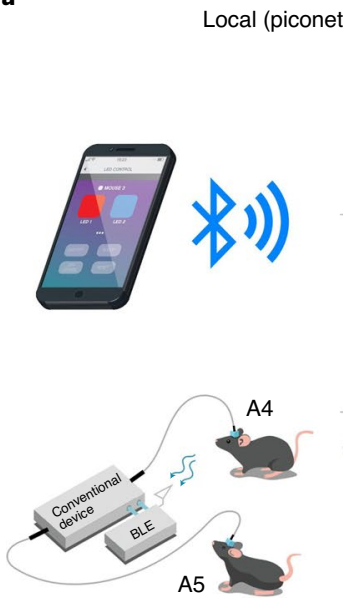

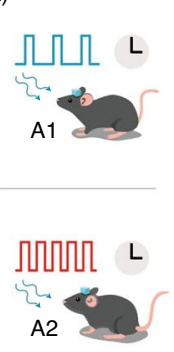
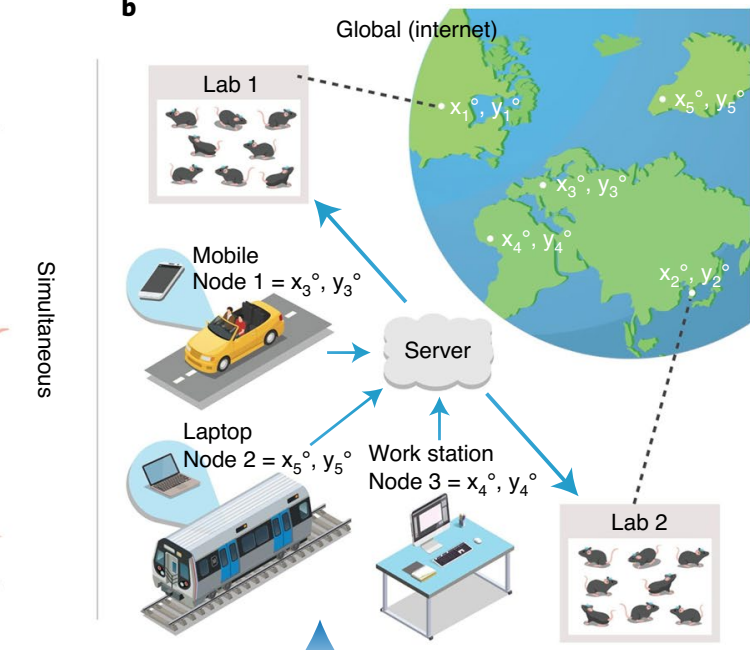

c

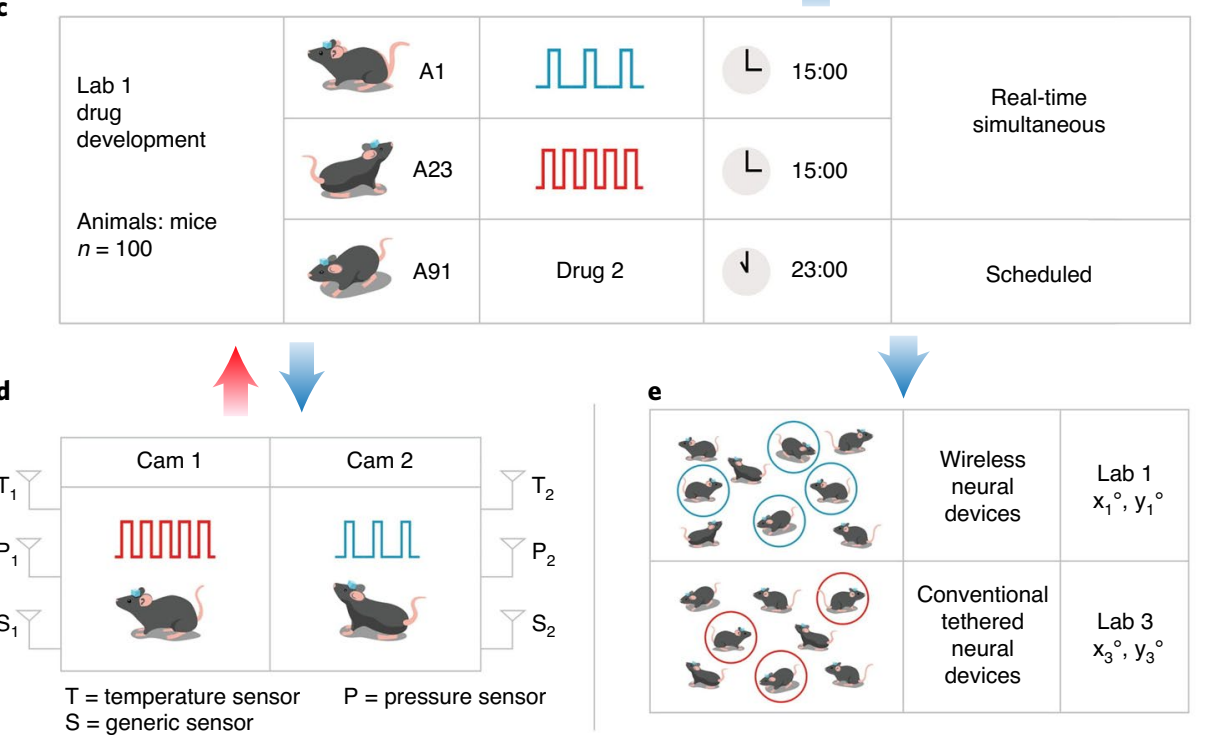

Fig. 1 | Concept and operation principle of WNBN for high-throughput in vivo neuroscience research. a,b, Remote control modes (local vs global) for WNBN control. For local wireless control (a), a commercial smartphone helps form an individual BLE piconet network, which allows communication with multiple devices simultaneously and/or selectively in its vicinity $(<100 \mathrm{~m})$. For global Internet control (b), remote researchers can use any device with an Internet connection to access the custom WNBN server, which is hosted by a local minicomputer inside each laboratory. Multiple users at multiple distinct locations can remotely and simultaneously communicate with various WNBN-enabled devices deployed in multiple laboratories around the globe. Both local (a) and global (b) control schemes can be further extended to conventional tethered equipment by connecting them to off-the-shelf BLE modules. c, Device triggering modes (real-time vs scheduled) for WNBN control. The WNBN Cloud allows multimodal and simultaneous control of neural devices either in real time or scheduled to a specific time in the future. Irrespective of the triggering modes, all controls are selective (controlling a specific group of animals within a large cohort) and simultaneous (control multiple target animals simultaneously). d, Schematic diagram illustrating bidirectional control and sensing capability, which allows not only sending control signals to various neural implants and laboratory tools, but also receiving data from various sensors and feedback systems. Automatic periodic gathering of data from the animal implants and/or laboratory tools can provide insightful information on animal experiments. e, Schematic diagram illustrating capability of selective large-scale control, which allows simultaneous and independent manipulation of various types of neural devices (that is, both emerging wireless and conventional tethered devices) within limited laboratory spaces for high-throughput neuroscience research.

(Extended Data Fig. 1), in comparison to contemporary technologies such as $\mathrm{RF}^{8-11,13,22,23}$ or IR technologies ${ }^{7,24}$, which do not have selective capabilities, leading to potentially limitless inaccurate pairings. Moreover, the communication of RCC with a specific RCM in a local piconet network is completely independent of its communication with other RCMs in the network. We leveraged this ability to enable the user to have complete control of which specific devices to manipulate simultaneously within a group, as well as which target functionalities to implement within each selected device. For example, with this feature, a single RCC can send multiple unique commands to 4 specific RCMs $(1,5,10,15)$ simultaneously within a piconet network of $15 \mathrm{RCMs} \mathrm{-} \mathrm{for} \mathrm{instance,} \mathrm{release} \mathrm{of} \mathrm{a} \mathrm{drug} \mathrm{using} \mathrm{a}$ microfluidic neural device implanted in mouse 1 (RCM 1), optogenetic excitation using a conventional tethered optical fibre implanted in mouse 5 (RCM 5), triggering of a laboratory centrifuge (RCM 10) and simultaneous delivery of light and drug using an optofluidic device implanted in mouse 15 (RCM 15). Hence, the selective and simultaneous control capability of local piconet WNBN technology enables versatile operation not only at device level (devices within a group), but also at functional level for independent control of each 
Table 1 | Comparison of wireless control schemes

\begin{tabular}{|c|c|c|c|c|c|c|}
\hline \multirow[t]{2}{*}{ Control Scheme } & \multirow{2}{*}{$\begin{array}{l}\text { Infrared (IR) } \\
\text { control }^{7}\end{array}$} & \multirow{2}{*}{$\begin{array}{l}\text { High frequency } \\
\text { (HF) control }\end{array}$} & \multirow{2}{*}{$\begin{array}{l}\text { Ultrahigh } \\
\text { frequency (UHF) } \\
\text { control }^{10,11}\end{array}$} & \multirow{2}{*}{$\begin{array}{l}\text { Single Bluetooth } \\
\text { control (S) })^{19,20}\end{array}$} & \multicolumn{2}{|l|}{ WNBN Control } \\
\hline & & & & & $\begin{array}{l}\text { Local piconet } \\
(P=n \times S)\end{array}$ & $\begin{array}{l}\text { Remote Internet } \\
(I=n \times P)\end{array}$ \\
\hline $\begin{array}{l}\text { Wireless access } \\
\text { scheme }\end{array}$ & $\begin{array}{l}\text { Infrared } \\
\text { one-to-one control }\end{array}$ & $\begin{array}{l}\text { HF broadcasting } \\
\text { control }\end{array}$ & $\begin{array}{l}\text { UHF } \\
\text { broadcasting } \\
\text { control }\end{array}$ & $\begin{array}{l}\text { Local BLE } \\
\text { one-to-one control }\end{array}$ & $\begin{array}{l}\text { Local one-to-many } \\
\text { BLE piconet network }\end{array}$ & Internet + BLE \\
\hline Access point & $\begin{array}{l}\text { Lightweight } \\
\text { custom } \\
\text { remote-control } \\
\text { module }\end{array}$ & $\begin{array}{l}\text { Bulky and } \\
\text { expensive RF } \\
\text { equipment }\end{array}$ & $\begin{array}{l}\text { Bulky and } \\
\text { expensive RF } \\
\text { equipment }\end{array}$ & $\begin{array}{l}\text { Any commercial } \\
\text { smartphone } \\
\text { (readily available) }\end{array}$ & $\begin{array}{l}\text { Any commercial } \\
\text { smartphone } \\
\text { (readily available) }\end{array}$ & $\begin{array}{l}\text { Any system with a } \\
\text { browser } \\
\text { (device independent) }\end{array}$ \\
\hline Access range & $\sim 1 \mathrm{~m}$ (low) & $<0.3 \mathrm{~m}$ (low) & $\begin{array}{l}\sim 0.1-0.2 \mathrm{~m} \\
\text { (very low) }\end{array}$ & $\begin{array}{l}\text { Local ( }<100 \mathrm{~m} \text {, } \\
\text { high) }\end{array}$ & $\begin{array}{l}\text { Local }(<100 \mathrm{~m} \text {, } \\
\text { high) }\end{array}$ & $\begin{array}{l}\text { Global } \\
\text { (very high) }\end{array}$ \\
\hline Access capability & $\begin{array}{l}\text { Any IR device } \\
\text { within range and } \\
\text { line of sight }\end{array}$ & $\begin{array}{l}\text { Multiple RF } \\
\text { devices within } \\
\text { range and line of } \\
\text { sight }\end{array}$ & $\begin{array}{l}\text { Multiple RF } \\
\text { devices within } \\
\text { range and line of } \\
\text { sight }\end{array}$ & $\begin{array}{l}\text { Any Bluetooth } \\
\text { device within range } \\
\text { (no line of sight } \\
\text { handicap) }\end{array}$ & $\begin{array}{l}\text { Multiple Bluetooth } \\
\text { devices in range } \\
\text { (no line of sight } \\
\text { handicap) }\end{array}$ & $\begin{array}{l}\text { Large-scale loT even } \\
\text { at far-away remote } \\
\text { locations } \\
\text { (no line of sight or } \\
\text { range handicap) }\end{array}$ \\
\hline Control capability & $\begin{array}{l}\text { Neither selective } \\
\text { nor simultaneous }\end{array}$ & $\begin{array}{l}\text { Limited } \\
\text { selectivity and } \\
\text { simultaneity }\end{array}$ & $\begin{array}{l}\text { Limited } \\
\text { selectivity and } \\
\text { simultaneity }\end{array}$ & Selective only & $\begin{array}{l}\text { Selective and } \\
\text { simultaneous }\end{array}$ & $\begin{array}{l}\text { Selective and } \\
\text { simultaneous and/or } \\
\text { scheduled }\end{array}$ \\
\hline $\begin{array}{l}\text { Selective simultaneous } \\
\text { device control } \\
\text { throughput }\end{array}$ & $\begin{array}{l}1 \\
\text { (neural implant) }\end{array}$ & $\begin{array}{l}1 \\
\text { (neural implant) }\end{array}$ & 1 & $\begin{array}{l}1 \\
\text { (neural implant) }\end{array}$ & $\begin{array}{l}15 \\
\text { (neural implants } \\
\text { and/or conventional } \\
\text { device) }\end{array}$ & $\begin{array}{l}\text { Scalable to hundreds } \\
\text { (neural implants } \\
\text { and/or conventional } \\
\text { devices) }\end{array}$ \\
\hline Multiple user control & No & No & No & No & No & Yes \\
\hline $\begin{array}{l}\text { Scalable closed-loop } \\
\text { control }\end{array}$ & No & No & No & No & Yes & Yes \\
\hline $\begin{array}{l}\text { Automation and } \\
\text { scheduling }\end{array}$ & No & No & No & No & No & Yes \\
\hline User interface & $\begin{array}{l}\text { Simple tactile } \\
\text { switches }\end{array}$ & $\begin{array}{l}\text { Complex control } \\
\text { switches }\end{array}$ & $\begin{array}{l}\text { Complex control } \\
\text { switches }\end{array}$ & $\begin{array}{l}\text { Universal } \\
\text { smartphone-based } \\
\text { system }\end{array}$ & $\begin{array}{l}\text { Universal } \\
\text { smartphone-based } \\
\text { system }\end{array}$ & $\begin{array}{l}\text { Universal } \\
\text { webpage-based } \\
\text { system }\end{array}$ \\
\hline Reliability & None & None & None & $\begin{array}{l}\text { Confirmation with } \\
\text { log tracking }\end{array}$ & $\begin{array}{l}\text { Confirmation with } \\
\text { log tracking }\end{array}$ & $\begin{array}{l}\text { Confirmation with log } \\
\text { tracking }\end{array}$ \\
\hline $\begin{array}{l}\text { On-demand } \\
\text { experimental setup } \\
\text { customizability }\end{array}$ & No & No & No & Yes & Yes & Yes \\
\hline Trigger latency & - & - & - & $\begin{array}{l}\text { Lower } \\
(<0.5 \mathrm{~ms})\end{array}$ & $\begin{array}{l}\text { Lower } \\
(<0.5 \mathrm{~ms})\end{array}$ & $\begin{array}{l}\text { Higher } \\
(<200 \mathrm{~ms})\end{array}$ \\
\hline $\begin{array}{l}\text { Special control setup } \\
\text { cost }\end{array}$ & $\begin{array}{l}\text { Low } \\
(<\$ 50)\end{array}$ & $\begin{array}{l}\text { High } \\
(\sim \$ 10,000)\end{array}$ & $\begin{array}{l}\text { High } \\
(\sim \$ 10,000)\end{array}$ & $\$ 0$ & $\$ 0$ & $\begin{array}{l}\text { Low } \\
(\sim \$ 5)\end{array}$ \\
\hline Setup time and effort & Moderate & High & High & Low & Low & Low \\
\hline Energy cost & Lowest & High & High & Low & Low & Moderate \\
\hline
\end{tabular}

IoT, Internet of Things.

individual feature or modality within a device (for control of multimodal devices). The local piconet WNBN can be easily established and controlled using a readily available smartphone and experiments can be rapidly scaled to several devices with minimal setup time and effort.

However, for control over very large-scale experiments across tens or hundreds of animal subjects and equipment or devices within a laboratory, the local piconet control starts reaching its access and scaling limit. To address this limitation, we implemented the scalable Internet control scheme by establishing a star network of multiple local piconet WNBNs, each with multiple wireless receivers (RCMs), connected to a single remotely located transmitter (RCC) (Fig. 2a(iii)). This scheme allows the Internet control to scale across various RCM devices within a laboratory and across multiple laboratories to enable seamless collaboration within or among research groups around the globe (Fig. 2b(iii)). Each RCC in this case can be globally accessed to control multiple piconet networks over the Internet. We used a local minicomputer (Raspberry Pi 3 Model B Quad-Core Broadcom 64bit ARMv8 1.2 GHz, Raspberry Pi Foundation; Supplementary Fig. 2) with the Internet access as an RCC, which communicates the data to multiple BLE dongles (BLED112, Silicon Labs), each of which then transfers the data wirelessly across local RCMs in the vicinity over BLE. The global Internet WNBN control network demonstrates similar selective and simultaneous capabilities (using BLE protocols) as that of local piconet WNBN. By increasing the number of BLE dongles in the minicomputer, the synchronized hopping and time division multiplexing techniques can be parallelized such that 
a
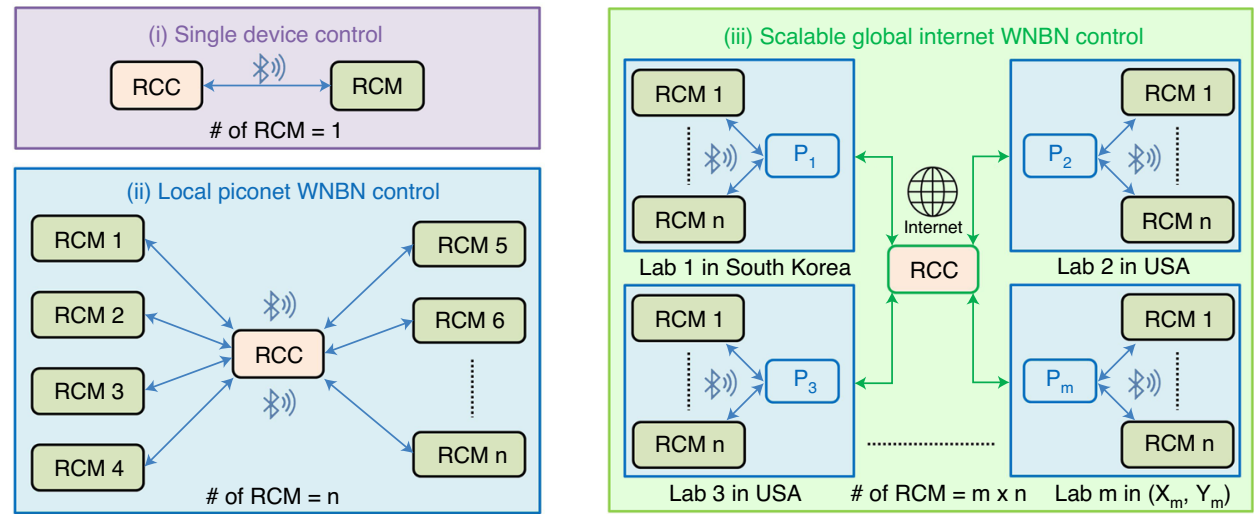

b

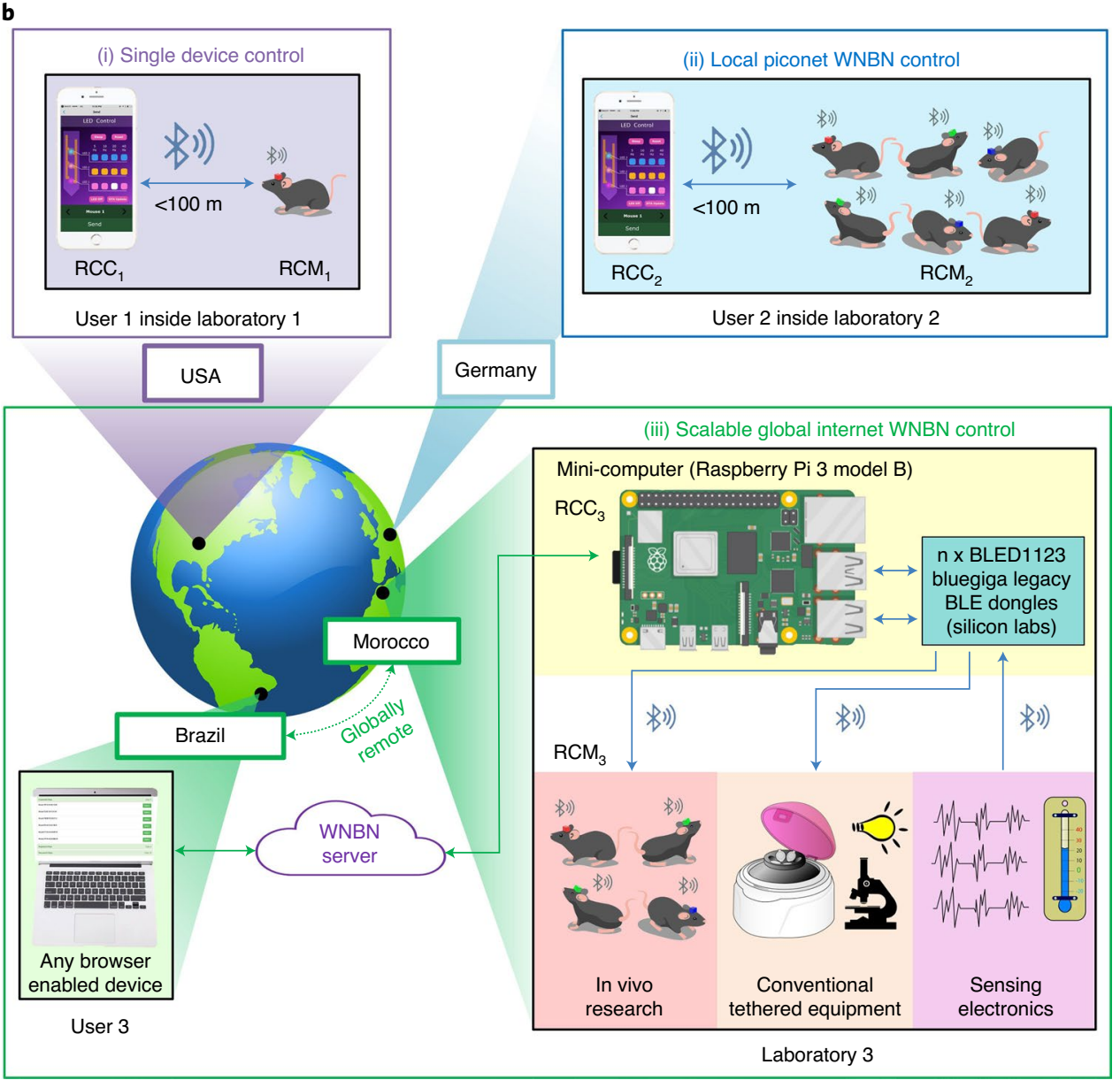

Fig. 2 | The WNBN architecture and signal flow. a, Block diagram of network topologies to highlight topological differences and signal flow between (i) conventional Bluetooth single device control and (ii,iii) WNBN multidevice control modes, where RCC, RCM and P stand for remote control centre, remote control module and piconet, respectively; (ii) local piconet WNBN control through a single RCC and (iii) global Internet WNBN control through multiple piconet networks $\left(P_{1}, P_{2}, P_{3}, \ldots\right.$, and $\left.P_{m}\right)$, all of which are connected to a single RCC. $\mathbf{b}$, Illustration of capabilities and access range of different technologies. (i) Single device control, where 1 RCC can control only 1 RCM at a time in its vicinity using BLE; (ii) local piconet WNBN mode, where a single RCC can selectively and simultaneously control up to 15 independent RCMs within its vicinity using BLE; and (iii) global Internet WNBN mode, where a single remotely located RCC can selectively and simultaneously control multiple devices across the globe at different laboratories (equipped with a minicomputer loaded with a custom WNBN operating system) using a combination of Internet and BLE technologies. The WNBN technology can control not only multimodal neural implants, but also tethered conventional equipment while gathering data from multiple analogue and digital sensors at the same time.

each individual piconet sub-network can wirelessly, selectively and simultaneously control numerous devices at a time. The number of controllable devices scales by increasing the number of connected BLE dongles. Each individual BLE dongle can support up to eight connections. Our custom software driver quickly adapts data multiplexing algorithms and wireless data streaming on the basis of the number of dongles connected for efficient and reliable wireless communication to all end devices. Stimulation parameters for 
neuromodulation, sensing data obtained from animals or laboratories, or control signals for triggering of conventional tethered equipment such as lasers and imaging probes connected to a BLE module can be communicated over global WNBN control. After developing this WNBN ecosystem, we next sought to test its function in various behavioural neuroscience settings.

Reliability of WNBN systems for in vivo neuroscience. A wireless network ecosystem meant to enable remote neuroscience experimentation that reduces human interaction while maintaining oversight of the experiment must be rapid (millisecond timescale) and reliable. To ensure quality and latency of data communication between the remote user and wireless networks, we validated the reliability of both the local piconet and the global Internet controls for control of both wireless neural devices and conventional neuroscience tools. First, we characterized and compared the WNBN control latencies for selective and simultaneous control of one or more outputs in a real-time or scheduled format. For these studies, we designed and employed a minimalistic, yet highly customizable and rechargeable wireless optogenetic probe (Fig. 3a and Supplementary Fig. 5) as an exemplary RCM device. This BLE-enabled device can be easily controlled using the WNBN ecosystem with minimal cost and effort, while parallel designs can be 3D-printed to further minimize costs ${ }^{25}$. This device was equipped with bilateral neural probes, where each probe housed two independently controlled microscale inorganic light-emitting diodes ( $\mu$-ILEDs) of different wavelengths $(470 \mathrm{~nm}$ and $589 \mathrm{~nm})$. For wireless control, it was then integrated with a BLE wireless module and a light battery $(0.3 \mathrm{~g}$; lithium polymer (LiPo) battery, PowerStream) through modular assembly.

Figure 3b,c, Extended Data Fig. 1 and Supplementary Videos 1-3 demonstrate the promptness and reliability of local piconet, and local and global Internet control modes for remote control of these wireless optogenetic devices (see Table 2 for summarized key performance metrics). Operation in all control modes is highly stable when a single RCM is paired to RCC, regardless of the distance between RCC and RCM (Extended Data Fig. 1a). These tests had zero failures (that is, $100 \%$ of success rate). Transmission was faster in the local piconet compared with the Internet controls (latency $\sim 20 \mathrm{~ms}$ for the piconet control versus $(\sim 55+\alpha)$ ms for the Internet control where $\alpha$ represents a signal propagation delay between the remote user and RCC; Fig. $3 \mathrm{~b}$ and Extended Data Fig. 1b). This increased latency for Internet control is mainly due to increased RCC-RCM distance, making the local piconet control more suitable for sophisticated experiments requiring higher temporal control resolution. For scheduled controls, RCC uses only the local network, thus the signal propagation delay can be completely eliminated. Therefore, using the scheduled control can make the latency difference between local and global Internet controls negligible. For simultaneous output or device control, the latency of additional devices receiving data increases since the frequency hopping algorithms need to find available frequencies for the additional devices (Fig. 3c). Overall, however, the differences are small and in most cases inconsequential. This is especially true for behavioural experiments with the time course of seconds or longer. Furthermore, the number of paired RCMs does not substantially affect the latency or success rate of BLE signal transmission (Extended Data Fig. 1c,d), except in the case of the local piconet where the number of allowable connections is limited to 15 by a smartphone. These results demonstrate the functionality and scalability of the WNBN control in reliably controlling numerous devices on millisecond timescales.

While benchtop testing provides clear performance hallmarks, integration with real neuroscience experiments requires proof-of-principle in vivo testing. To do so, we used multiple established animal behaviour models to test integration of WNBN infrastructure with in vivo neuroscience experiments. To demonstrate the effectiveness of WNBN-controlled manipulation, we used a well-validated feeding assay in which agouti-related protein (AgRP) expressing neurons were stimulated to drive food consumption in ad libitum fed conditions ${ }^{26,27}$. First, we implanted BLE-enabled blue $\mu$-ILED devices lateral to the paraventricular nucleus of the hypothalamus $(\mathrm{PVH})$ in $\operatorname{agrp}^{\mathrm{Cre}} \mathrm{x} \mathrm{Ai} 32$ mice. These mice have Cre-dependent expression of the blue-light sensitive cation channel, channelrhodopsin-2 (ChR2) within AgRP-expressing neurons (Fig. 3d). Following recovery, ad libitum fed mice were given access to a food pellet $(\sim 3 \mathrm{~g})$ for $3 \mathrm{~h}$ (Fig. $3 \mathrm{e}$ ) and received $1 \mathrm{~h}$ of $20 \mathrm{~Hz}$ photostimulation in an off-on-off manner. Blue-light photostimulation wirelessly activated via local piconet (Fig. 3f) or Internet WNBN control (Fig. 3g) significantly increased food consumption above baseline levels, validating the reliability of both WNBN control modes for wireless neuromodulation. Importantly, WNBN control not only works with these specialized BLE-enabled wireless neural devices (Fig. 3a), but can also be used with conventional laboratory equipment (Fig. 3h). Following the same experimental protocol, we used the Internet WNBN control to deliver commands $(20 \mathrm{~Hz}$ photostimulation) to typical transistor-transistor logic input/output ports on a diode-pumped solid-state (DPSS) laser tethered to fibre-optic-implanted mice, to drive the same increase in feeding behaviour (Fig. 3i,j). These proof-of-principle experiments verify that both control modes (that is, local piconet and Internet controls) of the WNBN system are equally reliable and highly versatile, allowing remote control of both wireless neural devices as well as conventional laboratory tools. This flexibility in downstream RCM targets optimizes laboratory adoptability while minimizing laboratory costs and setup efforts.

Simultaneous and selective control in WNBN systems for high-throughput neuroscience experimentation. To demonstrate the principles of WNBN control systems for multiple simultaneous experiments, we simultaneously targeted BLE-enabled blue $\mu$-ILED devices to the secondary motor cortex (M2) of six mice expressing ChR2 under the Thy1 promoter (Fig. 4a) ${ }^{28}$. Here, following a baseline exposure to an open arena, smartphone-based local piconet control was used to simultaneously photostimulate $(20 \mathrm{~Hz}) \mathrm{M} 2$ to induce locomotor behaviour (Fig. $4 \mathrm{~b}$ and Supplementary Video 4). This activation increased total locomotor activity (Fig. 4c), as well as induced rotation behaviour in a time-locked fashion (Fig. 4d). This local piconet-based control is the basis for expansion to scheduled and global control schemes. Using the unique identification of each BLE-enabled device in the WNBN, we can now program more complex interactive experiments, such as those including social interaction and/or those requiring selective control of discrete device features.

To demonstrate this independent selectivity, we used multicolour optogenetics to bidirectionally control the midbrain dopamine system to modulate social interaction ${ }^{29}$. Here we injected the ventral tegmental area (VTA) of dopamine active transporter Cre mice (DAT ${ }^{\mathrm{Cre}}$ ) with viral constructs containing soma-targeted blue-light-sensitive inhibitory opsin stGtACR2 ${ }^{30}$ and red-light-sensitive excitatory opsin Chrimson ${ }^{31}$. We then implanted bilateral, dual $\mu$-ILED (amber/blue) devices (Fig. 4e-g) directed towards the VTA. Following recovery, we selectively activated specific LEDs to target the distinct opsins during a test of social interaction (Fig. $4 \mathrm{~h}$, left). Using amber light to activate Chrimson (Fig. 4h, right), we demonstrated that DAT ${ }^{\text {Cre }}$ mice have increased social interaction with non-cagemate 'stranger' mice during VTA-dopamine neuron stimulation (paired $t$-test, $P<0.01$ ). In contrast, blue-light-mediated inhibition of dopamine neuronal activity through stGtACR2 stimulation (Fig. 4i) reduced social interaction, but did not reach significance $(P=0.08)$. Importantly, this simultaneous selective approach is not limited by device or species. To demonstrate this flexibility, we implanted Sprague-Dawley rats with microfluidic probes $^{19}$ (Extended Data Fig. 2a) directed 
a

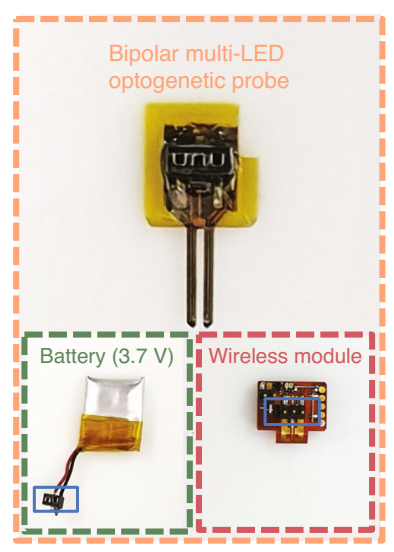

b

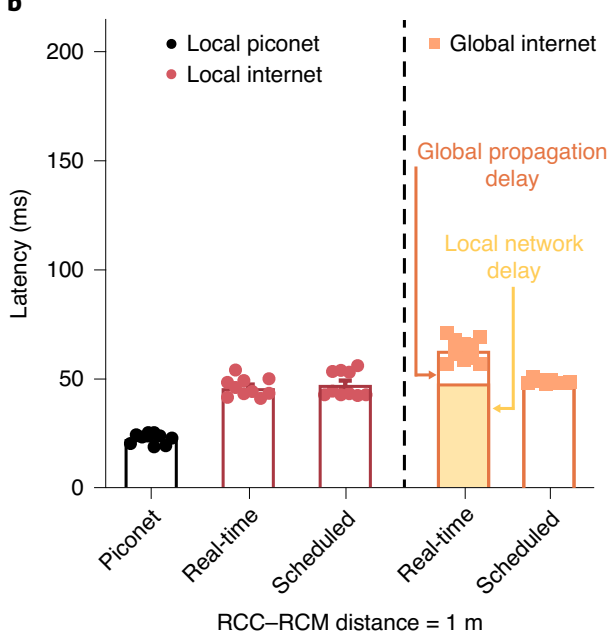

e

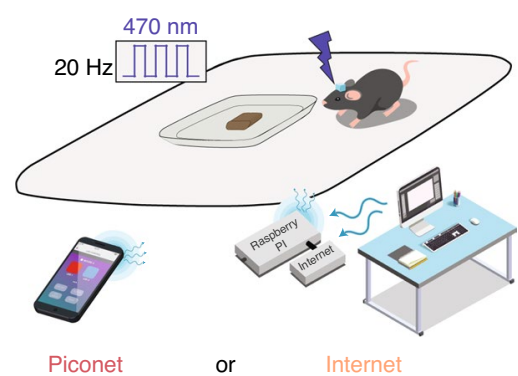

c

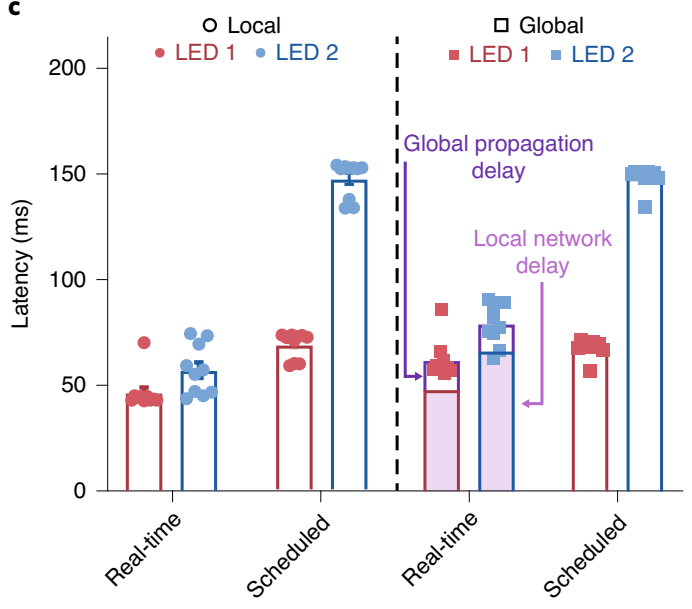

RCC-RCM distance $=1 \mathrm{~m}$ d

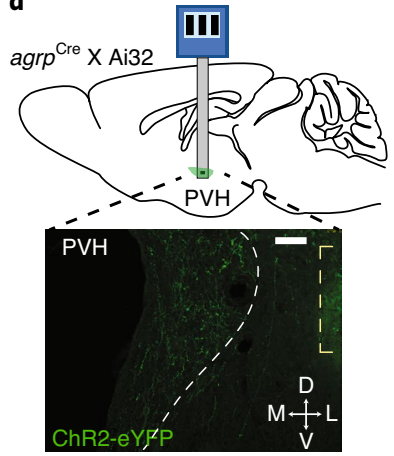

f

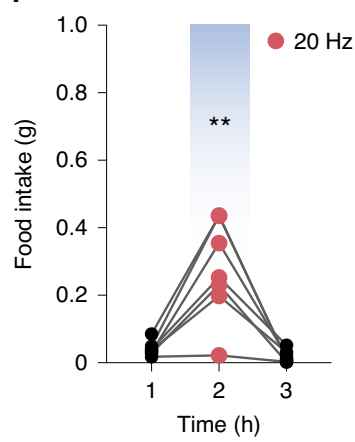

g

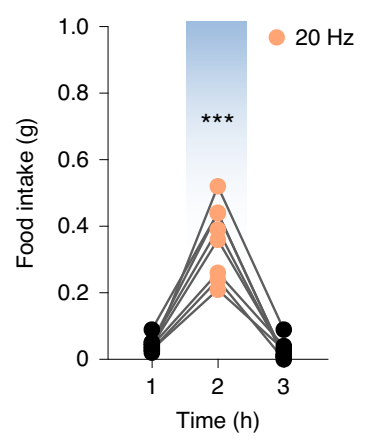

h

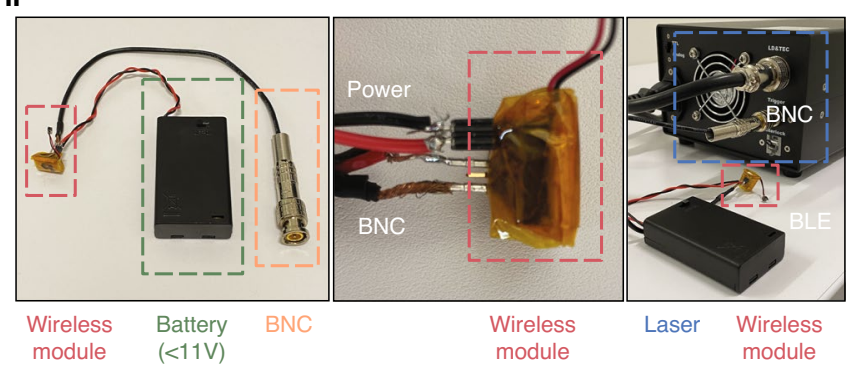

i

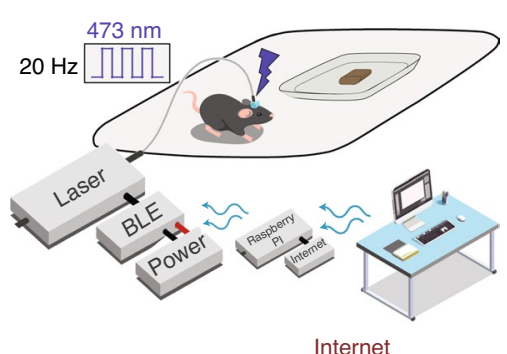

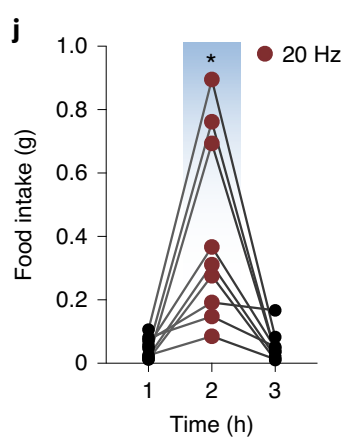

Fig. 3 | Implementation of local and global WNBN controls for in vivo neuroscience experimentation. a, Three major components of the modular and customizable wireless optogenetic system: a rechargeable LiPo battery, a soft $\mu$-ILED probe and a programmable wireless BLE module (RCM). b, Latency of

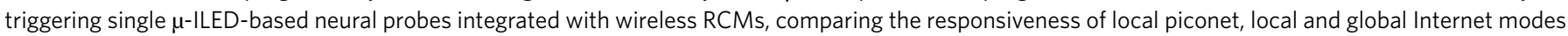
$(n=10)$. Distance between RCC and RCM is $1 \mathrm{~m}$. c, Latency of simultaneous triggering of dual $\mu$-ILEDs in optogenetic neural probes integrated with wireless RCMs, comparing the responsiveness of local and global Internet modes $(n=10)$. Orange arrow in $\mathbf{b}$ and dark purple arrow in $\mathbf{c}$ indicate a global signal propagation delay, which is dependent on the inter-distance between the remote user and RCC (for example, $15 \mathrm{~ms}$ delay between Boulder, CO and St. Louis, MO, USA). Note that this propagation delay can be ignored in scheduled controls. Distance between RCC and RCM is $1 \mathrm{~m}$. d, Top: sagittal brain cartoon of implantation of $\mu$-ILED device into the PVH of agrp ${ }^{\text {Cre }} \times$ Ai32 mice. Bottom: representative coronal image showing ChR2-eYFP expression (green)

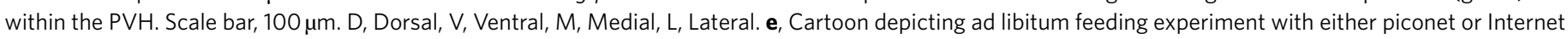
control. $\mathbf{f}, \mathbf{g}$, Amount of food consumed during local piconet-controlled (f) and local Internet-controlled ( $\mathbf{g}$ ) feeding of agrp ${ }^{\text {cre }} \times \mathbf{x}$ Ai32 mice, which consumed significantly more food during $20 \mathrm{~Hz}$ photostimulation compared with non-stimulation periods (1h) (repeated measures one-way ANOVA; (f) Hour 1 vs

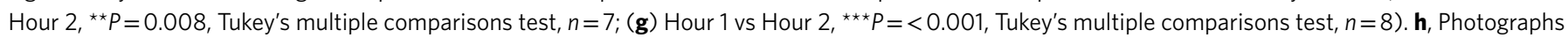
depicting set up of conventional device integration for Internet-controlled conventional optogenetic stimulation. BNC, Bayonet Neill-Concelman connector. i, Cartoon depiction of ad libitum feeding experiment using conventional optogenetic stimulation. j, Amount of food consumed during Internet-controlled

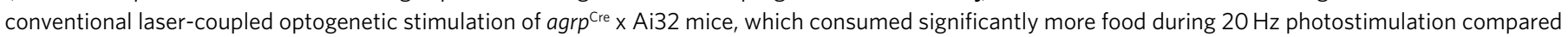
with non-stimulation periods ( $1 \mathrm{~h}$ ) (repeated measures one-way ANOVA, Hour 1 vs Hour $2,{ }^{\star} P=0.0126$, Tukey's multiple comparisons test, $n=9$ ).

at the nucleus accumbens and placed in a palatable food choice consumption assay. In this assay, local piconet WNBN-controlled microinfusion of mu-opioid receptor agonist, D-Ala2, NMe-Phe4,
Glyol5-enkephalin (DAMGO), drove binge-like food consumption for palatable high-fat diet as previously described ${ }^{32}$ (Extended Data Fig. 2b-e). 
Table 2 | Performance metrics of the WNBN system

\begin{tabular}{|c|c|c|c|}
\hline $\begin{array}{l}\text { WNBN } \\
\text { performance } \\
\text { metrics }\end{array}$ & $\begin{array}{l}\text { Local piconet } \\
\text { mode }\end{array}$ & $\begin{array}{l}\text { Local Internet } \\
\text { mode }\end{array}$ & $\begin{array}{l}\text { Global Internet } \\
\text { mode }\end{array}$ \\
\hline Latency & $\begin{array}{l}\sim 20 \mathrm{~ms} \\
\text { (maximum } \\
\sim 50 \mathrm{~ms} \\
\text { with } 15 \text { devices) }\end{array}$ & $\begin{array}{l}\sim 55 \mathrm{~ms} \\
\text { (maximum } \\
\sim 90 \mathrm{~ms} \\
\text { with } 22 \\
\text { devices) }\end{array}$ & $\begin{array}{l}(\sim 55+\alpha) \mathrm{ms} ; \\
\text { where } \alpha \text { is the } \\
\text { propagation delay } \\
\text { (maximum } \\
\sim(95+\alpha) \text { ms } \\
\text { with } 22 \text { devices) }\end{array}$ \\
\hline $\begin{array}{l}\text { Error rate } \\
\text { (misses/trials } \times \\
100 \text { ) }\end{array}$ & $\begin{array}{l}0 \% \\
\text { (with } \leq 15 \\
\text { devices) }\end{array}$ & $\begin{array}{l}<0.2 \% \\
\text { (regardless } \\
\text { of number of } \\
\text { devices) }\end{array}$ & $\begin{array}{l}<0.5 \% \\
\text { (regardless } \\
\text { of number of } \\
\text { devices) }\end{array}$ \\
\hline $\begin{array}{l}\text { Control distance } \\
\text { between user } \\
\text { and RCM }\end{array}$ & Local $(<100 \mathrm{~m})$ & No limit & No limit \\
\hline $\begin{array}{l}\text { Number of } \\
\text { controllable } \\
\text { devices }\end{array}$ & 15 & $\begin{array}{l}22+\text { with } \\
\text { multiple BLE } \\
\text { dongles } \\
\text { (100+ with } \\
\text { Bluetooth } \\
\text { mesh) }\end{array}$ & $\begin{array}{l}22+\text { with multiple } \\
\text { BLE dongles } \\
\text { (100+ with } \\
\text { Bluetooth mesh) }\end{array}$ \\
\hline $\begin{array}{l}\text { Power } \\
\text { consumption of } \\
\text { wireless module }\end{array}$ & $\begin{array}{l}\text { Tens of } \mathrm{mW} \\
\text { for } \mu \text {-ILED } \\
\text { operation }\end{array}$ & $\begin{array}{l}\text { Tens of } \mathrm{mW} \\
\text { for } \mu \text {-ILED } \\
\text { operation }\end{array}$ & $\begin{array}{l}\text { Tens of } \mathrm{mW} \\
\text { for } \mu \text {-ILED } \\
\text { operation }\end{array}$ \\
\hline Bandwidth & $\begin{array}{l}\sim 1 \mathrm{Mbs}^{-1} \\
\text { (BLE, extendable } \\
\text { up to } 2 \mathrm{Mbs}^{-1} \\
\text { for Bluetooth 5) }\end{array}$ & $\begin{array}{l}\sim 1 \mathrm{Mbs}^{-1} \\
\text { (BLE, extendable } \\
\text { up to } 2 \mathrm{Mbs}^{-1} \\
\text { for Bluetooth 5) }\end{array}$ & $\begin{array}{l}\sim 1 \mathrm{Mbs}^{-1} \\
\text { (BLE, extendable } \\
\text { up to } 2 \mathrm{Mbs}^{-1} \text { for } \\
\text { Bluetooth 5) }\end{array}$ \\
\hline
\end{tabular}

To demonstrate the WNBN's high-throughput capability to control systems for simultaneous and parallel experimentation, we conducted two concurrent locomotor and food consumption experiments. Here, Thy $1^{\text {ChR2-YFP }}$ mice $(n=8)$ were targeted with fibre optic implants for BLE-enabled control of conventional DPSS $473 \mathrm{~nm}$ laser stimulation of the M2 (Fig. $5 \mathrm{a})^{28}$. In addition, $\operatorname{agrp}^{\mathrm{Cre}} \mathrm{X}$ Ai32 mice $(n=12-13)$ were targeted with BLE-enabled blue $\mu$-ILED devices for the homecage stimulation of the PVH to drive feeding behaviour (Fig. 5b). Following recovery from surgery, ad libitum fed $\operatorname{agrp} p^{\mathrm{Cre}} \times \mathrm{Ai} 32$ mice were trained to collect pellets from a feeding experimentation device version 3 (FED3) ${ }^{33}$ overnight for $3 \mathrm{~d}$. FED3 is an open-source homecage feeding device that allows continuous food intake measurements inside a standard mouse homecage with minimal experimenter intervention. Following this training period, all animals from both experiments were placed in separate staging areas within the behavioural testing room - small open fields for the Thy $1^{\text {ChR2-YFP }}$ mice and FED3-enabled home cages for the agrp ${ }^{\text {Cre }}$ $\mathrm{x}$ Ai32 mice $-1 \mathrm{~h}$ before simultaneous testing. This coordinated experiment began by giving agrp ${ }^{\mathrm{Cre}} \mathrm{x} \mathrm{Ai} 32$ mice access to FED3 pellet dispensers (20 mg per pellet) (Fig. $5 \mathrm{c}$ ). During the first hour of FED3 access, Thy $1^{\text {ChR2-YFP }}$ were connected to fibre optic patch cables and allowed to acclimate for $10 \mathrm{~min}$. The experimenter then used the WNBN web interface to schedule a series of 'ON' and 'OFF' commands to be sent to all $21 \mathrm{BLE}$-enabled devices to initiate $20 \mathrm{~Hz}$ photostimulation in all mice. The first set of $21 \mathrm{ON}$ commands were sent at $60 \mathrm{~min}$. Following $5 \mathrm{~min}$ stimulation, $8 \mathrm{OFF}$ commands were sent to stop stimulation for the Thy ${ }^{\text {ChR2-YFP }}$. Fifty-five minutes later, 8 ON commands were sent to initiate a second Thy $1^{\text {ChR2-YFP }}$ stimulation time. Five minutes later, a final set of $21 \mathrm{OFF}$ commands were directed to all BLE-enabled devices to stop all stimulation in both experiments. This resulted in two 5 min stimulation periods for the Thy $1^{\text {ChR2-YFP }}$ mice and a single $1 \mathrm{~h}$ stimulation period for the $\operatorname{agrp} p^{\mathrm{Cre}} \mathrm{x}$ Ai32 mice (Fig. 5c). Internet-scheduled photostimulation significantly increased food pellet retrieval in $\operatorname{agrp}^{\mathrm{Cre}} \mathrm{x}$ Ai32 (Fig. 5d,g,i) mice while simultaneously increasing locomotion and rotation in Thy $1^{\text {ChR2-YFP }}$ mice (Fig. $5 \mathrm{~d}-\mathrm{f}, \mathrm{h}$ ). Individual heatmaps show simultaneous and distinct behavioural activation during these stimulation periods in each experiment (Fig. 5g,h). Continuous monitoring of pellet retrieval with the FED3 devices shows a leftward shift in the inter-pellet retrieval interval during photostimulation that persists during the hour following photostimulation, despite an overall reduction in pellets obtained during this time (Fig. 5j,k). One week following this test, we coordinated a second multibehaviour experiment to include locomotion and home-cage food consumption without the FED3 devices as described in Extended Data Fig. 3a. Again, scheduled and simultaneous photostimulation increased food consumption in $\operatorname{agrp}^{\mathrm{Cre}} \mathrm{x}$ Ai32 mice (Extended Data Fig. 3b,c,g) while concurrently increasing locomotion and rotation in Thy $1^{\text {ChR2-YFP }}$ mice (Extended Data Fig. $3 \mathrm{~d}-\mathrm{g}$ ). In both feeding experiments, photostimulation increased caloric intake; however, $\operatorname{agrp}^{\mathrm{Cre}} \mathrm{x}$ Ai32 mice had higher caloric intake during initial FED3 access (Hour 1) compared with free food access (Extended Data

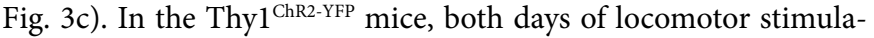
tion increased overall distance travelled and rotations; however, the second stimulation produced a larger relative response on the second test day, suggesting possible plasticity in response to repeated photostimulation (Extended Data Fig. 3e). Importantly, these simultaneous experiments were all conducted by a single experimenter controlling the experimental parameters through the WNBN web interface. This combination of experiments demonstrates the effectiveness of WNBN in simultaneously controlling specialized BLE-enabled wireless neural devices and conventional laboratory equipment to coordinate high-throughput experimentation.

Scaling wireless networks can introduce issues of network crowding and interference. While these two discrete and independent behavioural experiments were conducted twice within the same animal facility, this facility did not have excessive miscellaneous and unconnected Bluetooth devices within range of the minicomputer. At any given time, there were typically $\sim 10$ non-related devices representing BLE-enabled security cameras, smart building infrastructure, smartphones and computer peripherals. Increasing experimental scale to tens or hundreds of devices, however, could be limited due to signal interference among these and unconnected devices. One approach to limiting this interference would be to incorporate advanced Bluetooth mesh technology ${ }^{34}$ into the WNBN ecosystem. In a proof-of-principle benchtop experiment, we demonstrate this integration by enabling selective device activation of 82 devices over a physical distance of $4 \mathrm{~m}$ (Supplementary Video 5 and Fig. 6). This initial test suggests that advanced Bluetooth mesh technology is a feasible approach to limit Bluetooth signal interference in the WNBN ecosystem.

Automated control and data collection to reduce observer effects in behavioural neuroscience. In addition to on-demand device activation, delayed scheduling in the global Internet WNBN enables automated and semi-automated in vivo experimentation. Here, stimulation or monitoring protocols are established for future dates and times. These remote-control capabilities eliminate the need to be physically present in the laboratory, thereby alleviating some elements of an 'observer effect' ${ }^{1,5}$. As a versatile demonstration of this scheduled Internet-controlled stimulation, we used this approach to control laser-tethered fibre-optic-implanted mice in a typical optogenetics experiment. Here we tested whether the known arousal modulating properties of the locus coeruleus noradrenergic system ${ }^{11,35}$ extend to its projections into the medial prefrontal cortex (mPFC). To virally target this system, we used animals that exclusively express Cre recombinase in cells expressing dopamine beta hydroxylase $(D b h)$, the enzyme necessary for the conversion 

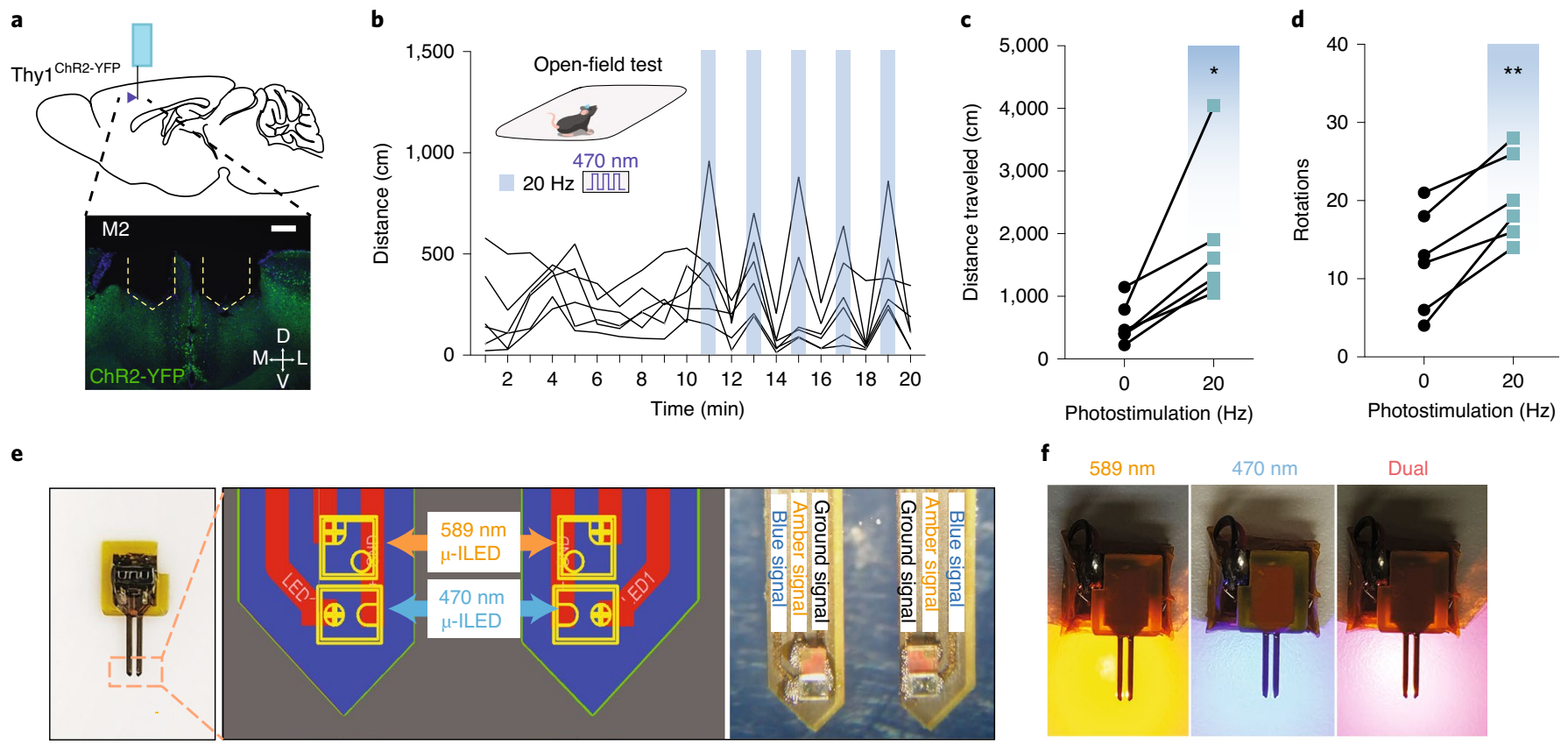

f $589 \mathrm{~nm}$
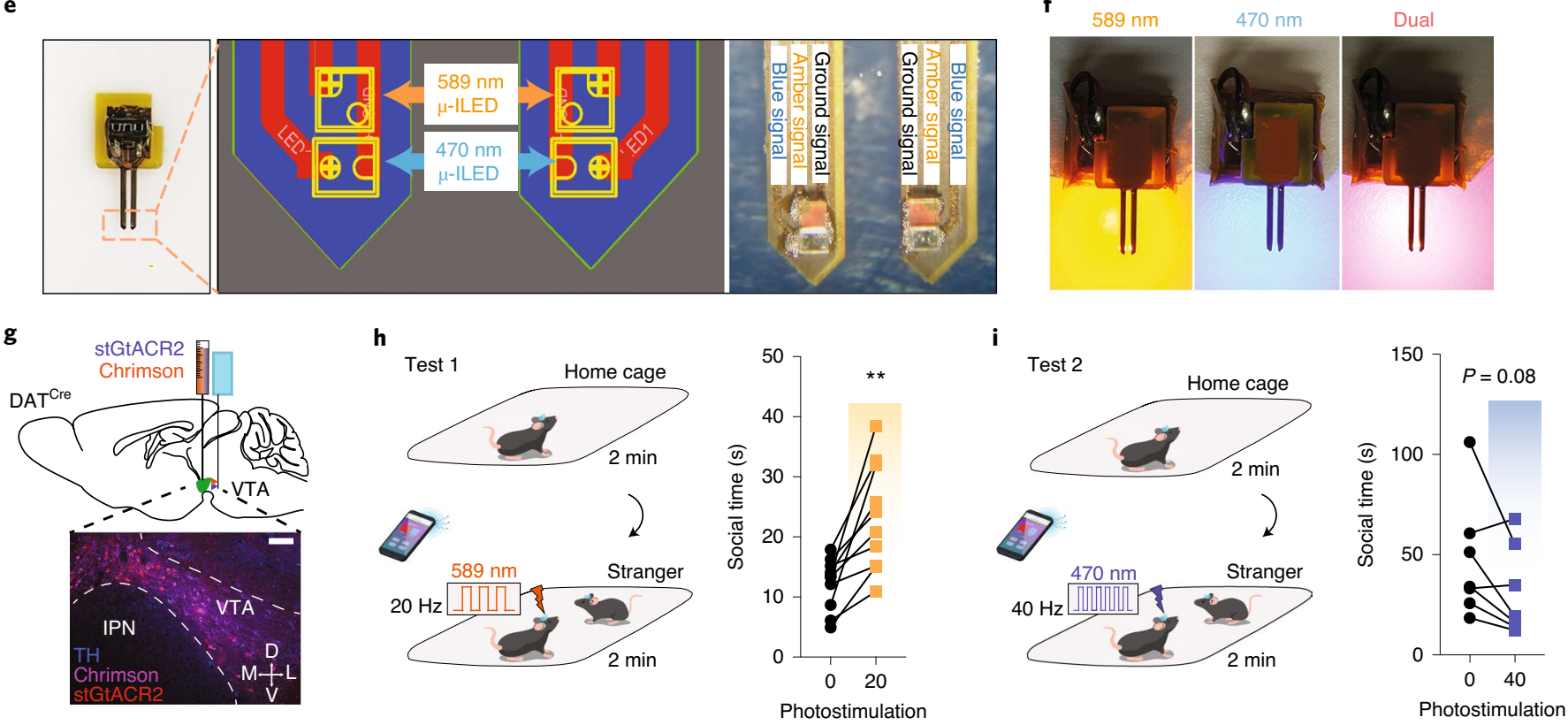

$(\mathrm{Hz})$

$(\mathrm{Hz})$

Fig. 4 | Simultaneous and selective in vivo control in WNBN-controlled systems. a, Top: sagittal brain cartoon of implantation of $\mu$-ILED device into the M2 of Thy ${ }^{1 h R 2}$ mice. Bottom: representative coronal image showing implant location and ChR2-YFP expression (green) within the cortex. Scale

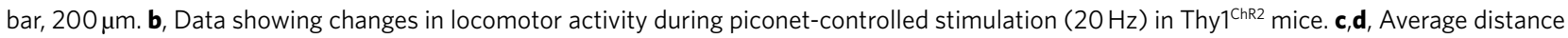
travelled (c) and average rotations (d) during local piconet-controlled stimulation intervals (c: two-tailed paired $t$-test $(0 \mathrm{vs} 20 \mathrm{~Hz})$, ${ }^{\star} P=0.0249, n=6$; d: two-tailed paired $t$-test ( 0 vs $20 \mathrm{~Hz}$ ), ${ }^{\star}{ }^{\star} P=0.003, n=6$ ). e, Photographs and cartoon diagram depicting design of a bipolar multi- $\mu$-ILED optogenetic probe. $\mathbf{f}$, Representative photographs of the wireless optogenetic device highlighting its ability to control individual $\mu$-ILEDs ( $589 \mathrm{~nm}, 470 \mathrm{~nm}$ and both, respectively). g, Top: sagittal brain cartoon depicting viral injection of AAV1-hSyn1-SIO-stGtACR2-FusionRed, AAV5-Syn-FLEX-Chrimson-tdTomato and bilateral $\mu$-ILED device implantation into the VTA of DATre mice. Bottom: representative coronal image showing immunohistochemistry for tyrosine hydroxylase (TH) (blue), stGtACR2 (red) and Chrimson (magenta) expression within the VTA. Dashed lines indicate boundaries of separate brain areas; VTA: ventral tegmental area, IPN: interpeduncular nucleus (Scale bar, $100 \mu \mathrm{m})$. $\mathbf{h}$, Left: cartoon depicting social interaction test with $20 \mathrm{~Hz}$ stimulation at $589 \mathrm{~nm}$ for Chrimson-mediated neuron excitation. Right: Chrimson stimulation $(20 \mathrm{~Hz})$ significantly increases social interaction in DATCre:Chrimson/GtACR2 mice (two-tailed paired $t$-test ( 0 vs $20 \mathrm{~Hz}$ ), $\left.{ }^{\star \star} P=0.0014, n=10\right)$. i, Left: cartoon depicting social interaction test with $40 \mathrm{~Hz}$ stimulation at $470 \mathrm{~nm}$ for stGtACR2-mediated neuronal inhibition. Right: this paradigm reduces social interaction in DATCre:Chrimson/GtACR2 mice (two-tailed paired $t$-test $(0$ vs $40 \mathrm{~Hz}$ ), $P=0.086, n=7$ ). Data are presented as mean \pm s.e.m. of biological replicates.

of dopamine to norepinephrine. $D b h^{\text {Cre }}$ mice were injected with AAV5-EF1 $\alpha$-DIO-ChR2-eYFP in the locus coeruleus and implanted with a chronic fibre optic ${ }^{36}$ in the medial prefrontal cortex (Fig. 6a). Following recovery, mice were placed in a behavioural test chamber and allowed to acclimate for $24 \mathrm{~h}$. The experimenter scheduled two global Internet WNBN-controlled stimulations ( $5 \mathrm{~Hz}$ for $15 \mathrm{~min}$ ) via the web interface to occur following acclimation (Fig. 6b). The next day, early light phase (AM) scheduled stimulation increased locomotor activity in $D b h^{\text {Cre }}$ animals (Fig. 6c), but late light phase (PM) stimulation had no clear effect (Fig. 6d). This suggests that activation of this pathway promotes general arousal that may be circadian rhythm dependent. Importantly, as these experiments were scheduled to occur automatically and without experimenter intervention, we found a strong negative correlation between baseline locomotion and activity during the photostimulation (Fig. 6e). Notably, the remote access capability of the global Internet WNBN can also be used to log data from selected sensing hardware to obtain vital data on laboratory conditions, such as temperature, humidity, air pressure, or any other sensor with an integrated BLE module (Fig. 6f). Here we used inexpensive off-the-shelf hardware - Simblee breakout board (RFD77101 Breakout Module, Simblee) and atmospheric sensor (Environmental Sensor BME680, SEN-16466, Sparkfun) 
to detect mouse home-cage temperatures for $24 \mathrm{~h}$ (Fig. 6f,g). We remotely detected changes in cage temperature for cages containing standard group-housed mice and empty control cages. Mouse cage temperatures increased during the $12 \mathrm{~h}$ 'night', while 'day' temperatures remained relatively stable (Fig. 6h,i). These data could provide reasonably inexpensive and reliable measures of overall cage activity $^{37,38}$. Critically, this technology could be expanded to include other behavioural and physiological detection systems as a means to automate, optimize or scale data collection while minimizing experimenter intervention.

\section{Discussion}

The WNBN system employed here gives the user the means to effortlessly scale and rapidly adapt to their needs or preference. This flexibility allows for selective large-scale control of BLE technology in behavioural neuroscience. This BLE-based system overcomes limitations of contemporary wireless neural devices that arise from inevitable non-selective batch triggering by their line-of-sight handicap ${ }^{7-11,13}$. Specifically, while most other approaches to wireless stimulation activate all nearby wireless devices in an identical manner, BLE can select each individual device or device feature for activation, and this software-based selectivity can be scaled with minimal effort, cost and hardware design considerations or setups. While many different schemes are available to make wireless devices for optogenetic manipulation, electrical recording and drug deliv$\mathrm{ery}^{7-9,11,13,15,23,24,39-41}$, the most advanced of these systems all rely on technology that cannot exclusively target an individual animal. In addition, there have been several efforts to send commands to perturb neural activity over the Internet ${ }^{42}$, but most of these have typically been pure demonstrations of feasibility rather than to improve experimental practice. One recent study sent commands from a smartphone over the Internet to provide optogenetic stimulation ${ }^{15}$; however, the presented technology likewise does not allow for individual animal and/or device function targeting or programmable operation to automate experiments. Compared with commercial high-throughput systems, which only allow a locally accessed (minimal wireless control), limited application (minimal data type versatility and compatibility) and limited scalability (limited number of multiplexed units), the WNBN ecosystem offers several unique advantages. The WNBN system provides a highly versatile and programmable universal wireless interface that can be globally accessed from anywhere and used with a diverse assortment of research laboratory equipment. Furthermore, this network ecosystem approach also allows multiplexed, real-time (or scheduled) and secure access to multiple remotely located laboratories around the globe. Table 1 and Supplementary Tables 1 and 2 detail the advantages and limitations of our approach compared with other relevant technologies.
While many capabilities of the local piconet WNBN mode and global Internet WNBN mode overlap at the microlevel, each technology offers unique tools and features for the scientific community to exploit on the basis of experimental scale, time and setup constraints. The local piconet WNBN mode enables rapid creation of local on-the-fly networks with minimal effort and complexity (by simultaneously controlling multiple unique experiments from the user's smartphone). This mode, however, requires users to be physically present in the laboratory and offers limited automation and scheduling. On the other hand, the global Internet WNBN mode can be used as a global collaborative tool across multiple laboratories, where researchers can potentially exploit its remotely controlled automation and scheduling capabilities. Remote access can be from the same user at home or in the office or from a different laboratory altogether. This global mode only requires minimal hardware in the form of a minicomputer as the server and any Internet browser-enabled device to access it. Furthermore, this global system is able to scale this level of network control across numerous simultaneously run experiments, thus carrying the potential to greatly enhance research throughput and progress. In particular, the global Internet WNBN can be customized to include any number of piconet networks within the ecosystem (by adding minicomputers at each location) and/or any number of devices within each piconet sub-network (by adding BLE dongles in the minicomputer). Overall, with minimalistic and accessible hardware infrastructure, both local piconet and global Internet modes of WNBN enable large groups of selective and simultaneous, wirelessly and/or remotely controlled experiments on-the-fly with 100\% device target accuracy. With complete control over customization of stimulation and data acquisition parameters in real-time or scheduled fashion, WNBN technology can help boost research throughput by enabling automation of large-scale simultaneously run experiments.

While the principal control schemes are the same whether the WNBN is used on-the-fly with a smartphone or remotely and scheduled over the Internet, there are important technical and ethical considerations to take before implementation. For example, the local smartphone control is suitable for experiments where shorter control latency (that is, $20 \mathrm{~ms}$ ) is desired. On the other hand, the Internet control is more appropriate when a larger scale of animal experiments (that is, a few tens of animals or more) are required. Another important factor is the duration of the experiment and what the power needs are of the devices being controlled. For acute experiments, any of the battery systems presented here are sufficient. However, for longer and more chronic studies, expansion of the battery pack or conversion to an AC power supply may be necessary. Future innovations in wireless recharging of batteries will enable near limitless remote access to the BLE-based system.

Fig. 5 | Simultaneous WNBN control of high-throughput behavioural experimentation. a, Left: sagittal brain cartoon of implantation of fibre optic implant M2 on Thy ${ }^{\text {ChR2-YFP }}$ mice. Right: representative coronal image showing implant location and ChR2-YFP expression (green) within the cortex. Scale bar, $250 \mu \mathrm{m}$. b. Left: sagittal brain cartoon of implantation of $\mu$-ILED device into the PVH of agrp ${ }^{\text {Cre }} \times$ Ai32 mice. Right: representative coronal image showing ChR2-eYFP expression (green) within the PVH. Scale bar, $100 \mu \mathrm{m}$. c, Cartoons and timeline describing locomotion and ad libitum pellet feeding experiment with Internet-scheduled simultaneous control. Blue bar indicates duration of photostimulation. $\mathbf{d}$, Simultaneous behavioural outputs depicting locomotion $(\mathrm{m})$ for Thy $7^{\text {ChR2-YFP }}$ mice and pellets retrieved per minute in agrp ${ }^{\text {Cre }} \times$ Ai32 mice in 1 min bins during coordinated behavioural experimentation.

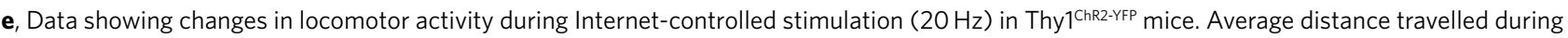
Internet-scheduled stimulation was significantly higher than at pre-stimulation and post-stimulation periods (repeated measures one-way ANOVA, for Stimulation 1: Pre1 vs Stim1, ${ }^{\star \star} P=0.0066$ and Stim1 vs Post1, ${ }^{\star} P=0.0443$, Tukey's multiple comparisons test, $n=8$; for Stimulation 2: Pre2 vs Stim2, ${ }^{\star}{ }^{*} P=0.0012$ and Stim2 vs Post2, ${ }^{\star} P=0.0115$, Tukey's multiple comparisons test, $\left.n=8\right)$. f, Rotations in Thy ${ }^{\text {ChR2 }}$ mice over 80 min $(1$ min bins) that includes two stimulation periods. $\mathbf{g}, \mathbf{h}$, Heatmaps depicting changes in behavioural activity for agrp ${ }^{\text {Cre }} \times$ Ai32 mice $(\mathbf{g})$ and Thy $1^{\text {ChR2-YFP }}$ mice (h) during coordinated scheduled photostimulation. $\mathbf{i}$, Average number of pellets retrieved during $1 \mathrm{~h}$ periods. Blue bar indicates the hour of $20 \mathrm{~Hz}$ stimulation. agrp ${ }^{\mathrm{Cre}} \times \mathrm{Ai} 32 \mathrm{mice}$ consumed significantly more pellets during $20 \mathrm{~Hz}$ photostimulation compared with non-stimulation periods (1h) (repeated measures one-way ANOVA, Hour 1 vs Hour $2,{ }^{\star \star} P=0.0048$ and Hour 2 vs Hour $3,{ }^{\star \star \star \star} P<0.0001$, Tukey's multiple comparisons test, $\left.n=12\right)$. j,k, Number of pellets (j) and kernel density estimate distributions $(\mathbf{k})$ of inter-pellet intervals during each $1 \mathrm{~h}$ period in agrp ${ }^{\mathrm{Cre}} \times \mathrm{Ai} 32$ mice. The area under each kernel density estimation curve is 1 and the area under any particular section of the curve estimates the probability of inter-pellet intervals of that duration occurring. Data are presented as mean \pm s.e.m. of biological replicates. 
The incorporation of wireless networks into biomedical sciences carries numerous ethical considerations for the health, safety and security of the research subjects involved. While our WNBN b

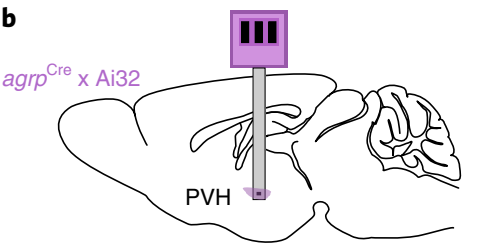

d

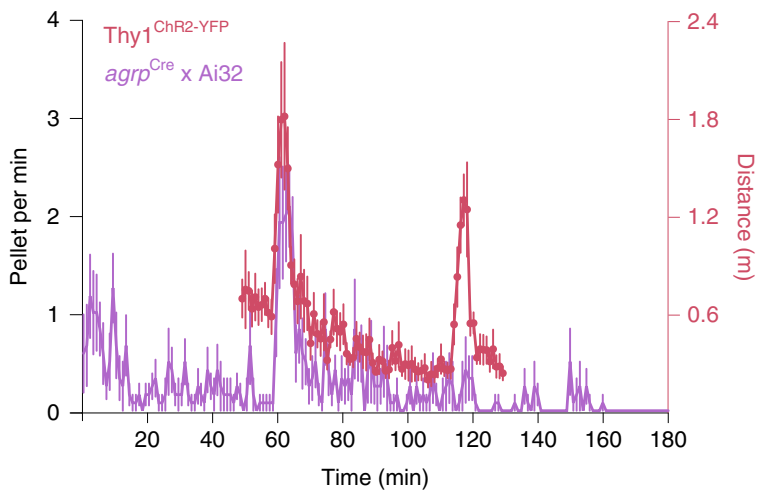

g
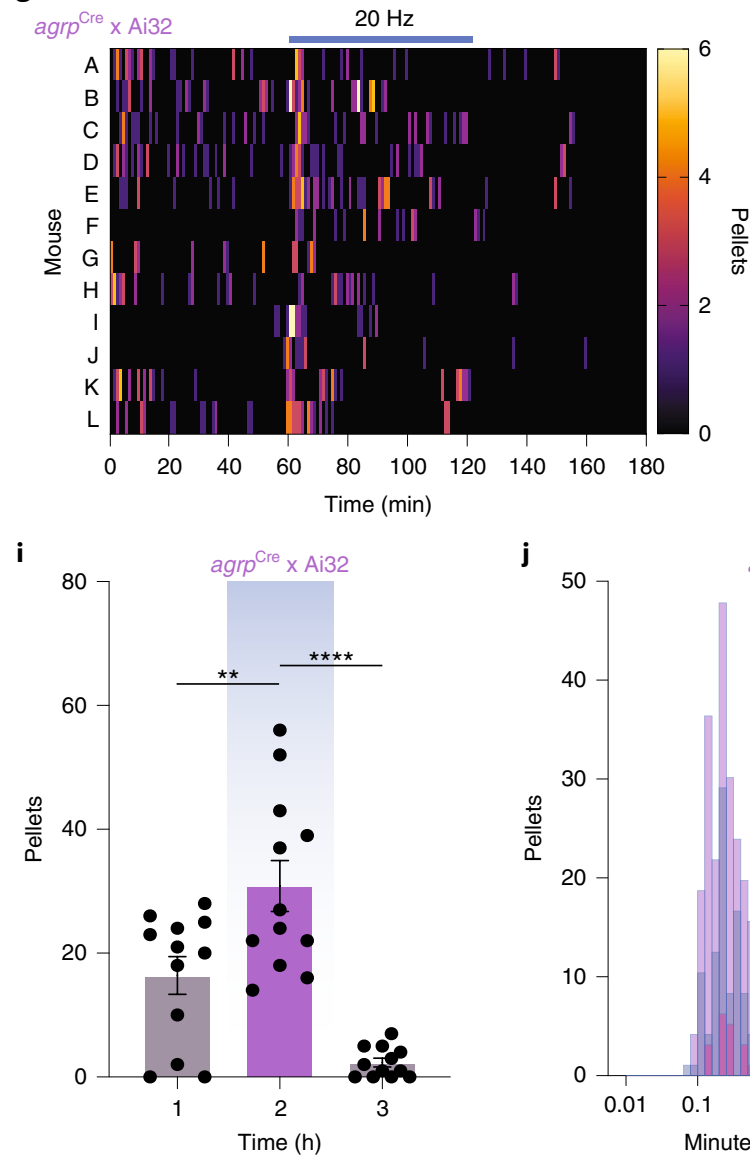

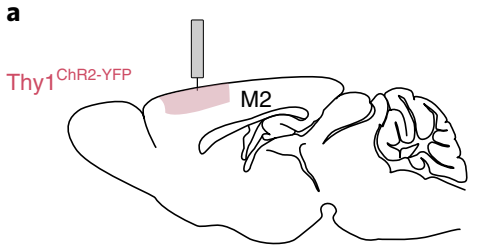

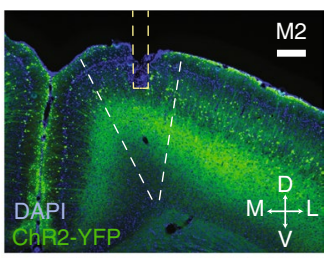

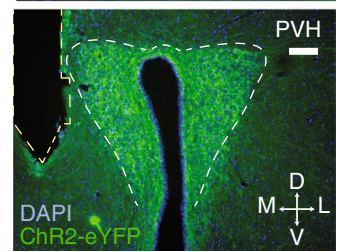

$\mathrm{PVH}$ system enables fully remote and global access, it is important to note that none of the experiments in this study were performed without the necessary human oversight to ensure animal safety. c
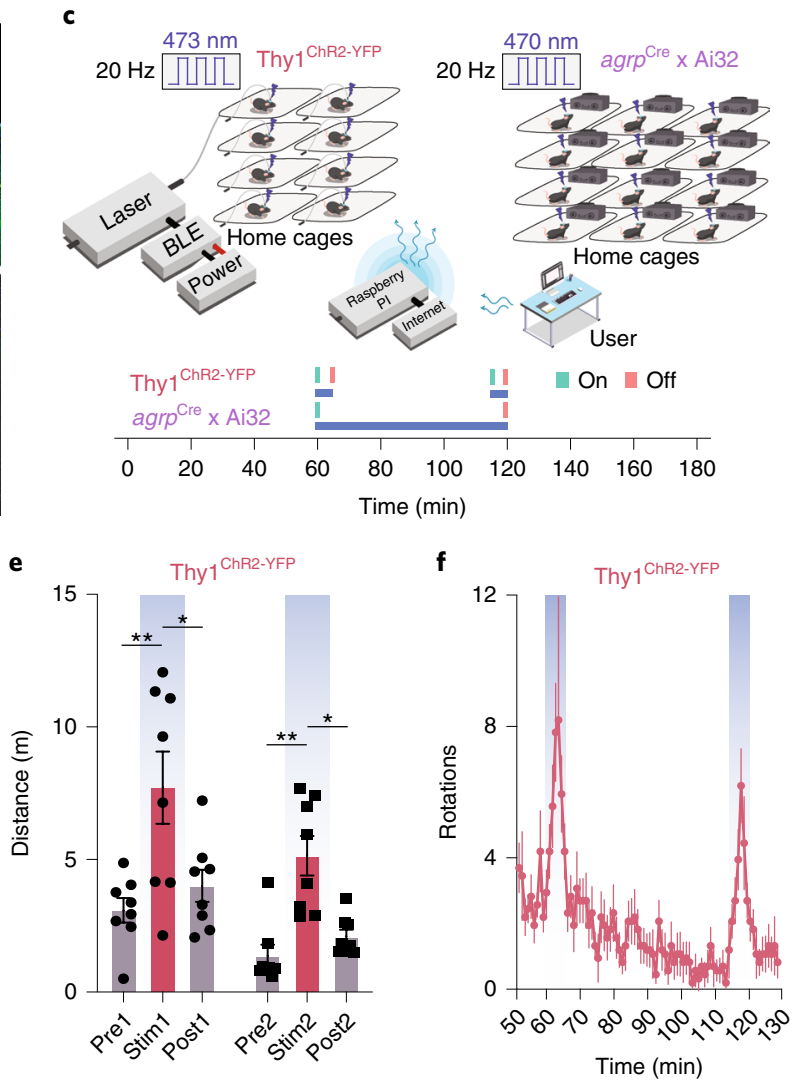

h
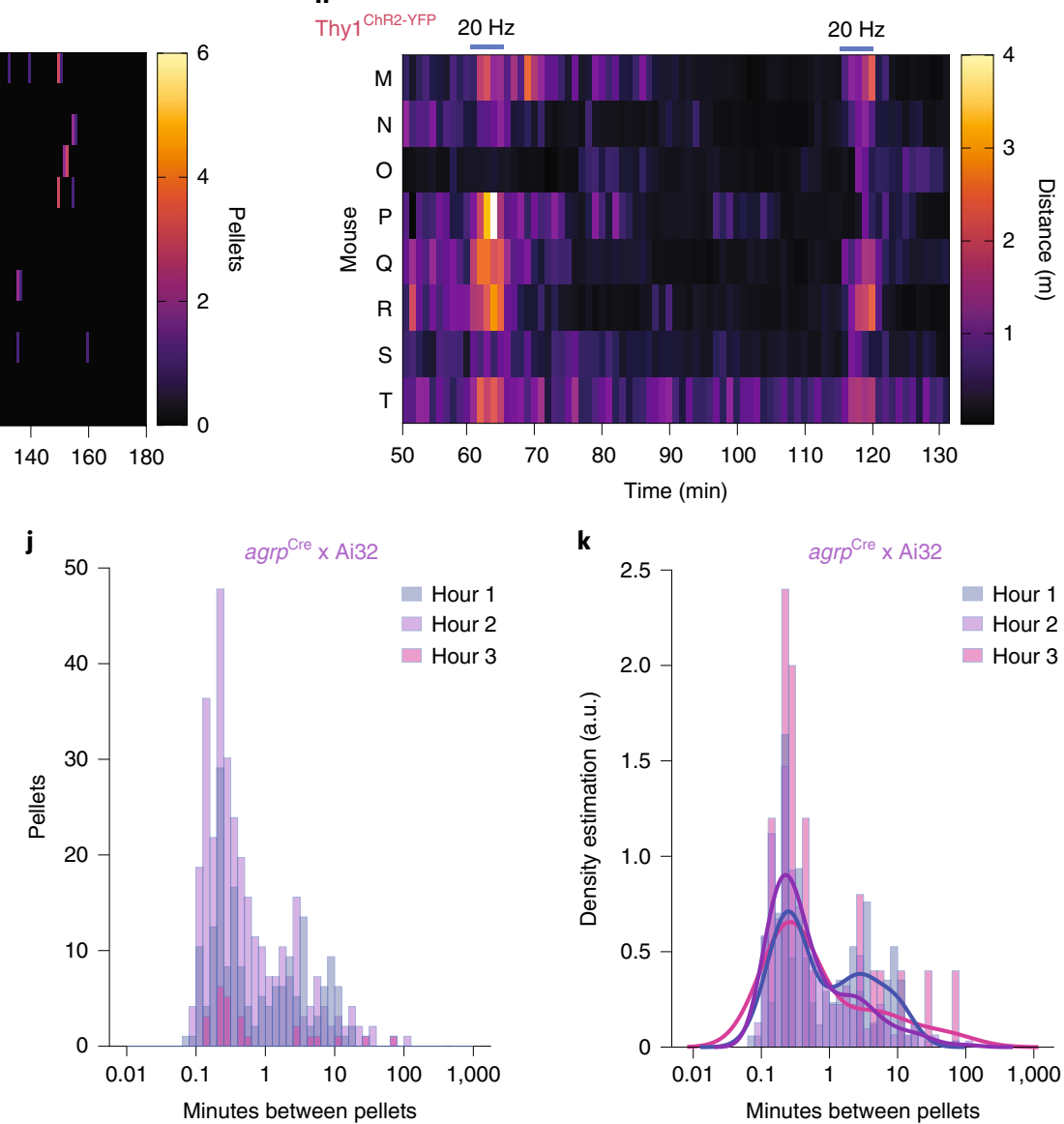


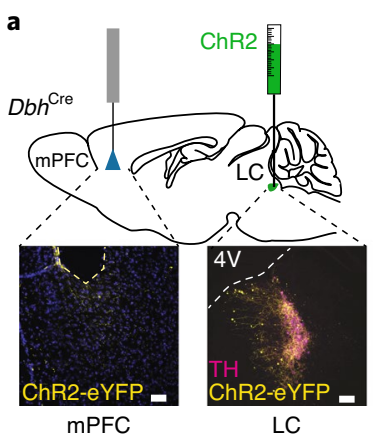

f

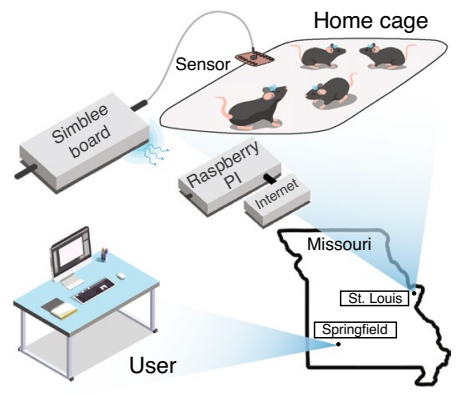

b
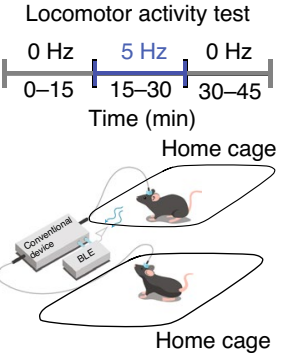

Home cage

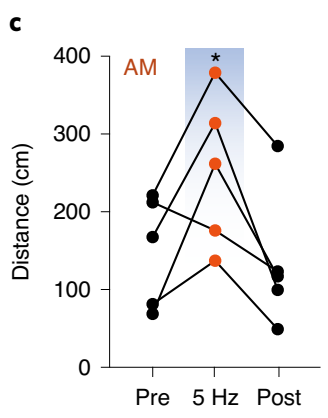

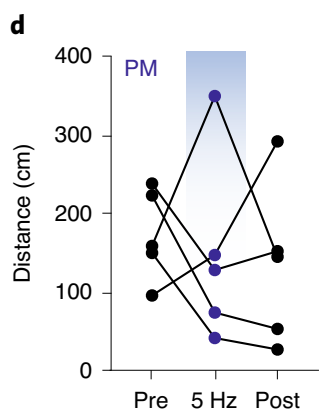

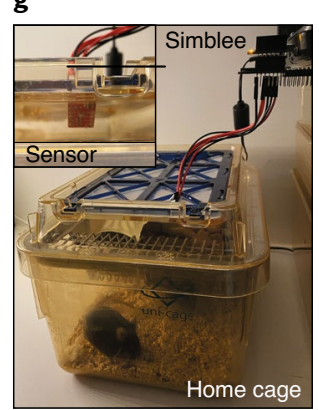

h

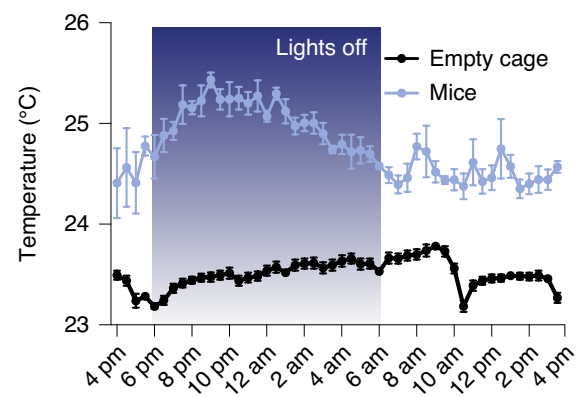

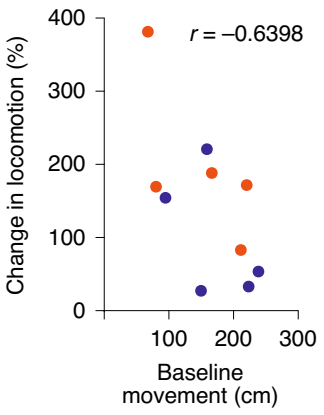

i

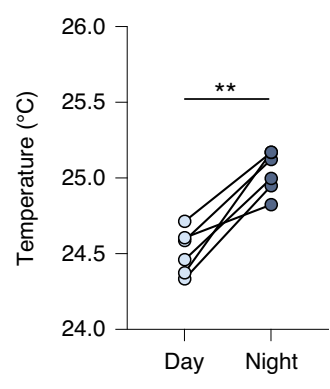

Fig. 6 | Conventional device integration for Internet-controlled experimentation and data collection. a, Top: sagittal brain cartoon depicting viral injection of AAV5-ef1 $\alpha$-DIO-ChR2-eYFP into the locus coeruleus (LC) and fibre optic implantation into the mPFC of $D b h^{\text {Cre }}$ mice. Bottom: representative coronal image showing immunohistochemistry for ChR2-eYFP expression within the mPFC and LC. Images show ChR2-eYFP (yellow) and tyrosine hydroxylase (TH) (pink) or Nissl (blue). Scale bars, $50 \mu \mathrm{m} ; 4 \mathrm{~V}$, fourth ventricle. b, Cartoon depicting implementation of scheduled global Internet control in conventional optogenetic stimulation to drive locomotor activity. c, $D b h^{\mathrm{CrelL} L \mathrm{mPFC}: \mathrm{ChR2}}$ mice had significantly higher locomotion during the AM $5 \mathrm{~Hz}$ stimulation compared with non-stimulation periods $\left(15 \mathrm{~min}\right.$ ) (repeated measures one-way ANOVA, $5 \mathrm{~Hz}$ vs Post, ${ }^{\star} P=0.0392$, Tukey's multiple comparisons test, $n=5$ ). d, No significant change was detected during the $\mathrm{PM} 5 \mathrm{~Hz}$ stimulation. $\mathbf{e}$, Baseline movement was negatively correlated with evoked locomotion ( $r=-0.6398, P=0.0464, n=10$ pairs). $\mathbf{f}$, Cartoon schematic of Simblee and sensor setup for home-cage temperature detection by remote user. In this case, as depicted in the cartoon, the commands were scheduled in Springfield, MO, USA for detection to occur in St. Louis, MO, USA. g, Photograph of Simblee board, sensor and mouse cage setup for monitoring cage temperature. Inset: photograph of sensor location inside mouse cage. h, Graph depicting average cage temperature collected over a $24 \mathrm{~h}$ period. Black circles indicate empty cage temperatures, blue circles indicate mouse cage temperatures. Dark bar indicates dark phase of light cycle (12h:12 h). Data are presented as mean \pm s.e.m. of biological replicates. $\mathbf{i}$, Graph depicting temperature change of mouse cage during dark phase of $12 \mathrm{~h}: 12 \mathrm{~h}$ light cycle (two-tailed paired $t$ test (Day vs Night), ${ }^{\star *} P=0.0011$ ). Data are presented as individual biological replicates.

While all optogenetic and pharmacological modifications were monitored closely by highly trained onsite personnel, the environmental sensing represents an opportunity to assess laboratory conditions remotely. This can be safely achieved remotely and this particular study was conducted from $\sim 350 \mathrm{~km}$ outside of the laboratory (Fig. $6 \mathrm{f}-\mathrm{i}$ ). However, because these studies took place in the home cage, animal welfare was ensured through daily animal husbandry health and safety checks, as with any other home cage in the animal colony. As researchers begin to incorporate wireless network ecosystems into their experimental workflows, it is absolutely necessary that investigators carefully vet the potential outcomes. If a neural manipulation is being operated remotely, appropriate safeguards need to be in place to mitigate any potential harm to the research subject. These considerations are similar to any home-cage-based study, such as those for circadian and feeding behaviours ${ }^{33}$ where experiments often occur over many hours with the experimenter in a separate location.

The WNBN system requires user credentials for access, and thus only authorized users can access and conduct experiments. The connection between the users and the WNBN system's webserver is encrypted using Hypertext Transfer Protocol Secure (HTTPS), so eavesdropping on the communication channel is not possible. Furthermore, the WNBN system can easily support Internet
Protocol Security (IPSec), creating strong site-level security by connecting a remote network to a user's local network. The Bluetooth communication between an RCC and RCMs is not encrypted since they are usually located nearby (a few metres) inside the protected lab facilities. However, care should be taken before granting authorization to new users of the WNBN. In particular, the WNBN can likely reduce or streamline skilled labour involvement with some research studies. For example, Fig. 5 and Extended Data Fig. 3 were both conducted by a single researcher - a feat that would have been difficult or impossible to do alone with the requisite temporal control. However, this should not mean that sophisticated and delicate animal experiments could be ported to less-trained individuals. The use of a wireless control ecosystem must be restricted to those with the necessary experience and training to ensure animal safety. While one can imagine cooperative arrangements to conduct studies globally in a safe and secure manner, this should never be done without the necessary safeguards for animal health and well-being. On that note, in addition to potentially reducing skilled labour hours, the WNBN also has the potential to reduce and refine animal studies ${ }^{43}$. Enabling long-term home-cage-based experiments should reduce the number of animals needed by increasing statistical power in within-subject designs. In this same vein, wireless home-cage experiments, especially those that allow animals to be 
studied without human interaction, should help reduce stress to the animal subjects inherent to behavioural testing.

The proof-of-principle behavioural experiments presented here demonstrate that the WNBN ecosystem is readily accessible to most behavioural and circuit neuroscience laboratories. Whether using custom-designed BLE-enabled optical probes and drug delivery devices or by integrating BLE control into conventional laboratory hardware, these approaches make semi-automated, high-throughput in vivo neuroscience possible. Future use of bidirectional communication within the WNBN ecosystem can enable fully or semi-autonomous closed-loop systems where inputs from sensors trigger subsequent commands from the system for quality control or experimental purposes. Further increasing the data transmission and storage capacities of the WNBN will also help integrate other technologies, such as wireless neural recording ${ }^{44}$ and $\mathrm{Ca}^{2+}$ imaging data ${ }^{45}$. Integration with physiological sensors for remotely accessible, large-scale and fully automated closed-loop systems neuroscience experiments will enable study of cell and circuit function in more naturalistic settings. Large-scale experiments with tens to hundreds of devices can be limited due to Bluetooth signal interference among the devices. This potential interference can be eliminated by adopting an advanced Bluetooth mesh technology ${ }^{34}$ into the current WNBN system, as shown in our proof-of-principle experiment (Supplementary Video 5 and Fig. 6). Furthermore, while we demonstrate use of the WNBN in home cage and social interaction behavioural experiments, overcoming the line-of-sight handicap seen in other technologies ${ }^{7-11,13}$ makes experiments in truly unconstrained environments possible. It is reasonable to imagine using the WNBN infrastructure in large or complex environments that more closely mimic the natural world ${ }^{46}$ or in rapidly moving, unstructured settings ${ }^{47}$.

The WNBN infrastructure is based on readily available consumer hardware. We proactively made the decision to create the BLE-based networks using inexpensive and accessible methods so that these systems could be widely adopted by the community. In contrast to earlier generations of wireless optogenetic systems ${ }^{8,913,23}$, this system can be used immediately in any laboratory with equipment that uses transistor-transistor logic. In other words, the WNBN can readily trigger DPSS laser stimulation, timestamp electrophysiology experiments, activate behavioural apparatuses, initiate drug or fluid delivery, and much more. The WNBN system is agnostic to its downstream components and could have as much utility in a chemistry laboratory as it does in a behavioural neuroscience laboratory. Therefore, with proper planning, its ability to schedule and remotely control laboratory equipment could help alleviate the burden of workflow disruptions, such as those currently occurring during the SARS-CoV-2 pandemic ${ }^{48}$. Even while laboratories are fully operational, the ability to conduct automated, hands-free experiments within vivarium home cages will increase the quality and quantity of neuroscience data by limiting the observer effect, maximizing data acquisition from animal subjects and reducing the physical workload of the experimenter.

\section{Methods}

Design and fabrication of $\mu$-ILED optical probes. Fabrication of ultrathin, flexible neural probes for photostimulation and drug delivery are detailed in our previous papers ${ }^{24,40}$. Here we employed a different approach to enable mass deployment of low-cost, scalable and easy-to-use optogenetic probes with $\mu$-ILEDs. The probes were designed with a printed circuit board (PCB) design software (Altium Designer 18, Altium Limited) and constructed using conventional PCB manufacturing process. The neural probes were built on $25-\mu \mathrm{m}$-thick polyimide sheets with double-side metallic copper coating. The top copper layer $(18 \mu \mathrm{m})$ was patterned to form electrodes and help conduct electrical signals, while the bottom copper layer $(18 \mu \mathrm{m})$ remained intact to make probes stiff enough for seamless injection into brain tissue without the need for a temporary injection assist needle commonly used for soft neural probes ${ }^{24,40}$. The sandwiched structures were laser-cut into thin probe needles. Subsequently, $\mu$-ILEDs with appropriate colours blue (TR2227, Cree) or orange (TCE10-589, Three Five Materials) - were soldered on the electrode pads using an ultra-fine T5 solder paste (SMDLTLFP10T5, Chip Quik). To prevent damaging of $\mu$-ILED probes by biofluid, the probes were coated with 7- $\mu$-thick Parylene C layer using a parylene deposition tool (PDS 2010 Labcoater 2, Speciality Coating Systems). Then, plug-n-play female rectangular electrical connectors (M50-3140345, Harwin) were soldered at the base of the probes for connection with BLE wireless control modules. Finally, the fabrication was completed by mounting the probes on 3D-printed holders for easy handing.

Setup of WNBN hardware infrastructure. The WNBN hardware ecosystem (Fig. 2b) consists of a network of wirelessly connected devices that can be remotely controlled from either inside of the laboratory or from a different part of the globe The end devices that are controlled are called RCMs, which help connect various neural implants and laboratory equipment wirelessly to the WNBN ecosystem. The control devices that manipulate multiple remotely located RCMs simultaneously and selectively are called RCCs. Both RCMs (wirelessly controlled devices) and RCCs (user end devices) form the key nodes in the hardware ecosystem of WNBNs, allowing researchers to scale their experimental networks and research throughputs.

$R C M$. Being one of the key constituents of the WNBN hardware ecosystem, RCM includes a customizable and rechargeable wireless BLE circuit (Fig. 3a and Supplementary Fig. 5) or conventional tethered tool integrated with off-the-shelf BLE plug-in (Fig. 3h). These RCMs are either integrated with stimulation devices (neural devices), sensing electronics (for example, temperature, pressure, humidity, etc.) or conventional tethered hardware (for example, fibre optical systems, drug delivery systems, centrifuges, etc.), thus empowering them with the ability to communicate wirelessly with the local piconet and global Internet networks.

RCC. The other key constituent of the WNBN hardware ecosystem is the RCC, which remotely controls multiple RCMs present inside the target laboratory. For local piconet control, RCC consists of a commercially available smartphone, which can communicate directly with multiple wireless RCMs and rapidly form on-the-fly wireless networks. However, for global Internet control, any device with a web browser and Internet connectivity (Wi-Fi, Ethernet, Cellular) can act as RCC and communicate remotely with RCMs through a custom-designed WNBN server, which is hosted by an off-the-shelf minicomputer (Raspberry Pi 3 Model B Quad-Core Broadcom 64 bit ARMv8 1.2 GHz, Raspberry Pi Foundation). The USB ports of the minicomputer are then plugged with BLE dongles (BLED112, Silicon Labs) that help communicate with multiple wireless RCMs selectively and simultaneously, thus forming versatile wireless control topologies.

Setup and control of WNBN software. The WNBN software is controlled using GUIs that are accessible through Bluetooth-enabled (for local piconet) and Internet-enabled (for global Internet) RCCs. These serve as front-end interfaces allowing users to define, configure and control multiple RCMs wirelessly. Furthermore, the WNBN control schemes and protocols are 'user-defined' (that is, they need appropriate decoding algorithms in RCM firmware), thus enabling very high versatility for researchers to define their own experiments (such as customizable strings with information on frequency, pulse width and periodicity of stimulation cycles for an optogenetic experiment).

Local piconet mode. For local piconet control, the custom GUI application for the RCC (smartphone with built-in BLE hardware, Supplementary Fig. 3) utilizes the pre-installed Bluetooth libraries, services and built-in wireless protocols to directly communicate with various RCMs in the vicinity. After receiving advertisement information of available RCMs, the user connects to a specific set of RCM devices, which allows him/her to get communication and control access to that specific group, thus enabling very high selectivity and accuracy.

Global Internet mode. For global control, a single RCC (any Internet-enabled device) irrespective of its geographic location can control a large number of RCMs at multiple coordinates around the globe. The global Internet mode does not require the researcher to be physically present in the vicinity of RCMs. Hence, after gaining access to a browser in any RCC and logging on to the WNBN server address, the user requires password-protected credentials and appropriate access privileges (given by the admin) to allow secure wireless login. Once logged in, the user can access the WNBN GUI (Supplementary Fig. 4), which can allow him/her to configure and control various configurations, data communications and logging features on the webserver. Compared to local control, the global control requires an additional minicomputer (in the vicinity of wireless RCMs), which helps host the webserver (on one side), as well as assists in communicating with end RCMs in the vicinity (on the other side). After the user gains access to the Cloud and sends appropriate configurations/data to send/receive to/from RCMs using the front-end GUI of the WNBN server, the minicomputer receives them over the Internet before decoding the data. Using the Bluetooth Generic Attribute Profile (GATT) library, its custom operating system then communicates all information with wireless RCMs through its USB ports, which are plugged with multiple BLE dongles. The back-end algorithms in the custom operating system of the minicomputer help parallelize (on the basis of priority) the data stream through multiple BLE 
dongles, which further enhance large-scale real-time control and overall wireless connectivity.

Experimental subjects. Adult (20-30 g) C57BL/6 J mice, DAT-IRES-Cre, Thy1-ChR2-YFP, Dbh-IRES-Cre and AgRP-IRES-Cre backcrossed to C57BL/6 J mice and bred to Ai32 mice were group-housed, given access to food pellets and water ad libitum, and maintained on a $12 \mathrm{~h}: 12 \mathrm{~h}$ light:dark cycle (lights on at 6:00). All mice were transferred to a facility within the laboratory after weaning and remained in the facility in the laboratory at least 1 week before surgery, after surgery and throughout the duration of the behavioural assays to minimize stress from transportation and disruption from foot traffic. All mouse procedures were approved by the Animal Care and Use Committee of Washington University and conformed to US National Institutes of Health guidelines. Five-week-old male Wistar rats arrived in the laboratory and were initially pair housed, with males and females in separate rooms. Animals remained in a climate-controlled room at $22^{\circ} \mathrm{C}$ and maintained on $12 \mathrm{~h}: 12 \mathrm{~h}$ dark:light cycle (lights off at 11:00), with ad libitium access to rat food (Purina 5008). Testing occurred during the dark cycle (11:00-13:00). All rat procedures were approved by the University of Missouri Institutional Animal Care and Use Committee guidelines and approved protocols At approximately 7 weeks age, all rats were separated into single housing and a PVC tubing section was provided in cages for environmental enrichment.

Stereotaxic virus injection and device implantation surgeries. Adult mice were acclimated to the holding facility for at least $7 \mathrm{~d}$. In all surgical procedures, mice were anaesthetized in an induction chamber ( $1 \%-4 \%$ isoflurane) and placed into a stereotaxic frame (Kopf Instruments, Model 1900) where they were maintained at $1 \%-2 \%$ isoflurane. For social interaction experiments, DAT Cre mice were injected bilaterally using a blunt needle (86200, Hamilton) at a rate of $100 \mathrm{nl} \mathrm{min}{ }^{-1}$ DAT $^{\text {Cre }}$ mice were injected in the VTA $(-3.15 \mathrm{~mm}(\mathrm{AP})$; $0.5 \mathrm{~mm}(\mathrm{ML})$; and -4.5 (DV), AP: anteroposterior, ML: mediolateral DV: dorsoventral) with a $350 \mathrm{nl}$ cocktail of AAV5-Syn-FLEX-Chrimson-tdTomato and AAV1-hSyn1-SIO-stGtAC R2-FusionRed viruses. Mice were allowed to recover for 5 weeks before implantation and behavioural testing, permitting optimal expression of the virus. For VTA cell body stimulation and inhibition, mice were implanted with bilateral implants with $2 \mu$-ILED directly posterior to the VTA at $-3.4 \mathrm{~mm}$ (AP); $+0.5 \mathrm{~mm}$ $(\mathrm{ML})$; and $-4.5 \mathrm{~mm}(\mathrm{DV})$. For arousal experiments, $D b h^{\mathrm{Cre}}$ mice were injected into the locus coeruleus ( $-5.4 \mathrm{~mm}(\mathrm{AP}) ;+1.25 \mathrm{~mm}(\mathrm{ML})$; and $-3.65(\mathrm{DV})$ ) with $200 \mathrm{nl}$ AAV5-efla-DIO-ChR2-eYFP. Mice were allowed to recover for 5 weeks before implantation and behavioural testing, permitting optimal expression of the virus. For mPFC stimulation, $D b h^{\text {Cre }}$ mice were then implanted with unilateral, fibre optic implants $^{36}$ directed at the $\mathrm{mPFC}(+2.0 \mathrm{~mm}(\mathrm{AP}) ;+0.3 \mathrm{~mm}(\mathrm{ML})$; and $-2.25(\mathrm{DV}))$. For feeding experiments, $\operatorname{Agr} p^{\mathrm{Cre}} \mathrm{x}$ Ai32 $2^{\mathrm{ChR} 2}$ mice were unilaterally implanted with BLE-enabled $\mu$-ILED devices directed at the PVH $(-0.82 \mathrm{~mm}(\mathrm{AP}) ;-0.5 \mathrm{~mm}$ (ML); and $-5.25 \mathrm{~mm}(\mathrm{DV}))$. For locomotion experiments, Thy $1^{\text {Chr2-YFP }}$ mice were either implanted bilaterally with BLE-enabled $\mu$-ILED devices (Fig. 4) into the motor cortex $(+1.0 \mathrm{~mm}(\mathrm{AP}) ; \pm 0.5 \mathrm{~mm}(\mathrm{ML})$; and $-0.5(\mathrm{DV}))$ or unilaterally with a fibre optic implant (Fig. 5). The implants were secured and affixed with dental cement (C\&B Metabond Adhesive Luting Cement, Parkell). All mice were allowed to recover for at least 1 week following implantation before behavioural testing. For the microfluidic drug delivery experiments, 8-week-old $( \pm 3 \mathrm{~d})$ Wistar rats were anaesthetized with isoflurane (Sigma Aldrich) and stereotaxically implanted with a custom microfluidic device that contained housing for drug cartridges, batteries and InfraRed or BLE-control systems ${ }^{19,24}$. The 4-channel microfluidic probe was placed directly into the nucleus accumbens (bregma: $+1.4 \mathrm{AP}, \pm 2.0 \mathrm{ML}$, $-5.3 \mathrm{DV})^{49}$. The housing was then anchored to the skull with stainless steel screws. Light curable resin (Lang Dental) and glue adhered the drug housing device to the surface of the skull. Following surgery, rats were monitored for 1 week before beginning behavioural testing.

Drugs. The $\mu$-opioid receptor agonist, D-Ala2, NMe-Phe4, Glyol5-enkephalin (DAMGO) (Research Biochemicals) was dissolved in sterile $0.9 \%$ saline at a concentration of $0.25 \mu \mathrm{g}$ per $0.5 \mu \mathrm{l}$.

Tissue processing. Unless otherwise stated, animals were transcardially perfused with $0.1 \mathrm{M}$ phosphate-buffered saline (PBS) and then $40 \mathrm{ml} 4 \%$ paraformaldehyde. Brains were dissected and post-fixed in $4 \%$ paraformaldehyde overnight and then transferred to $30 \%$ sucrose solution for cryoprotection. Brains were sectioned at $30 \mu \mathrm{m}$ on a microtome and stored in a $0.01 \mathrm{M}$ phosphate buffer at $4{ }^{\circ} \mathrm{C}$ before immunohistochemistry and tracing experiments. For behavioural cohorts, viral expression and optical fibre placements were confirmed before inclusion in the presented datasets.

Immunohistochemistry. Immunohistochemistry was performed as described in our previous studies ${ }^{6-8,11}$. In brief, mice were intracardially perfused with $4 \%$ paraformaldehyde, and then brains were sectioned $(30 \mu \mathrm{m})$ and placed in $0.1 \mathrm{M}$ phosphate buffer until immunohistochemistry. Free-floating sections were washed in $0.1 \mathrm{M}$ PBS for three $10 \mathrm{~min}$ intervals. Sections were then placed in blocking buffer $(0.5 \%$ Triton X-100 and 5\% natural goat serum in $0.1 \mathrm{M} \mathrm{PBS})$ for $1 \mathrm{~h}$ at room temperature. After immersion in blocking buffer, sections were placed in primary antibody (chicken anti-tyrosine hydroxylase,1:2,000, Aves Labs; rabbit anti-ChR2, 1:500, American Research Products) overnight at room temperature. After three 10 min washes in $0.1 \mathrm{M}$ PBS, sections were incubated in secondary antibody (AlexaFluor 488 goat anti-rabbit, AlexaFluor 594 or 633 goat anti-chicken, Life Technologies) for $2 \mathrm{~h}$ at room temperature, followed by another three $10 \mathrm{~min}$ washes in 0.1 M PBS. Later, sections were incubated in NeuroTrace (435/455 blue fluorescent Nissl stain, ThermoFisher Scientific) for $1 \mathrm{~h}$, followed by three $10 \mathrm{~min}$ washes in $0.1 \mathrm{M}$ PBS, then three $10 \mathrm{~min} 0.1 \mathrm{M}$ phosphate buffer washes. After immunostaining, sections were mounted and coverslipped with Vectashield HardSet mounting medium (Vector Laboratories) and imaged on a Leica DM4 P epifluorescence microscope.

Behavioural experiments. All behavioural experiments were performed within a sound-attenuated room maintained at $23^{\circ} \mathrm{C}$ at least 1 week after habituation to the holding room and the final surgery. For all experiments, mice were brought into the experimental behaviour space and allowed to acclimate for at least $30 \mathrm{~min}$. For all experiments, experimenters were blinded to mouse genotype or experimental manipulation. All pharmacological interventions (for example, agonists and antagonists) were randomized and counterbalanced via block randomization before experimentation. Sample size was estimated via power analysis $\left(\mathrm{G}^{\star} \text { Power } 3\right)^{50}$, with a power of 0.85 and a standard deviation of $15-20 \%$ of the mean. Lighting was stabilized at $\sim 250$ lux for all behaviours. Movements were video recorded via a Charge-Coupled Device (CCD) camera and analysed using Ethovision XT 13 (Noldus Information Technologies). At the end of each study, mice were perfused with $4 \%$ paraformaldehyde, followed by anatomical analysis to confirm injection sites and cell-type-specific expression.

Locomotion assay. Before locomotion testing, a Bluetooth-connected battery pack was attached to each Thy1-ChR2-YFP mouse $(n=5)^{28}$. Each animal was then placed into a square $\left(50 \times 50 \mathrm{~cm}^{2}\right)$ open-field enclosure. Locomotor activity was monitored and measured via a CCD camera using Ethovision 13. Animals were allowed to explore the open-field apparatus for $10 \mathrm{~min}$, after which a piconet-initiated Bluetooth signal was sent to the intracranial device implant to drive $470 \mathrm{~nm}$ photostimulation at $20 \mathrm{~Hz}$. Each period consisted of a $1 \mathrm{~min}$ photostimulation followed by a $1 \mathrm{~min}$ off duration, over a $10 \mathrm{~min}$ period. For the scheduled, simultaneous behavioural experiments, locomotion testing was done in home cages placed within sound-attenuated boxes and behaviour was recorded with a CCD camera. Animals were connected to a $473 \mathrm{~nm}$ laser via an optical fibre patch cable and allowed to acclimate to the environment for $10 \mathrm{~min}$, after which an Internet-scheduled Bluetooth signal was sent to a Bluetooth-enabled controller to drive $20 \mathrm{~Hz} 473 \mathrm{~nm}$ photostimulation from the laser. After $5 \mathrm{~min}$, a second Internet-scheduled signal was sent to stop the stimulation. Fifty minutes later, a second series of Internet-scheduled commands was coordinated to drive another 5 min stimulation. All locomotion was monitored for $90 \mathrm{~min}$

Food intake assay. The food intake study was performed as previously described $^{26,27,51,52}$. agrp-Cre ${ }^{\mathrm{ChR} 2} \times$ Ai32 mice (10- to 12 -week-old male mice, $n=7-9$, Fig. 3; $n=13$, Extended Data Fig. 3) were singly housed for at least 2 weeks following surgery and handled daily to ensure acclimation and minimize any stress response to experimental procedures. On test day, a Bluetooth-connected battery pack was attached to each animal's intracranial device implant. Animals were then placed in a large polycarbonate cage inside a sound-attenuated box (Med-Associates) and allowed to acclimate to the environment for $1 \mathrm{~h}$. After the $1 \mathrm{~h}$ acclimation, one pellet of food $(\sim 3 \mathrm{~g})$ was placed on a weigh boat fixed to the cage centre. Following $1 \mathrm{~h}$ of food access, the food was weighed and immediately returned to the cage centre. Next, mice received $470 \mathrm{~nm}$ photostimulation at $20 \mathrm{~Hz}$ for $1 \mathrm{~h}$. Mice were allowed access to the food for a $3 \mathrm{~h}$ period, with measurements taken at $1 \mathrm{~h}$ intervals. Mice with implantation or ChR2 expression outside the PVH were excluded from the group after post hoc examination of eYFP expression. All food intake measurements were randomized and blind to the experimenter.

Food intake assay monitored via FED3. This food intake study was performed similarly as the previous food intake experiment. agrp-Cre ${ }^{\mathrm{ChR} 2} \mathrm{x}$ Ai32 (10- to 12 -week-old male mice, $n=12$ ) were singly housed for at least 2 weeks following surgery and handled daily to ensure acclimation and minimize any stress response to experimental procedures. Before experimental manipulation, animals were trained to use the FED3 pellet dispenser overnight for $3 \mathrm{~d}$. This device allows for the continuous monitoring of food intake. Using the 'free feeding' mode, the FED3 presents one $20 \mathrm{mg}$ pellet, then dispenses a new pellet once the previous pellet has been removed. On test day, a Bluetooth-connected battery pack was attached to each animal's intracranial device implant. Animals were then placed in a polycarbonate cage and allowed to acclimate to the environment for $1 \mathrm{~h}$. After acclimation, the FED3 pellet dispenser was placed in the cage. Following $1 \mathrm{~h}$ of access, mice received an Internet-scheduled command for $470 \mathrm{~nm}$ photostimulation at $20 \mathrm{~Hz}$ for $1 \mathrm{~h}$. Mice were allowed access to the FED3 for a $3 \mathrm{~h}$ period, with continuous monitoring of each animal's behaviour.

Social interaction assay. Social interaction in the home cage was examined as described previously ${ }^{29}$. Briefly, DAT-Cre $\mathrm{GtACR} /$ Chrimson $^{\mathrm{G}}$ cage mates were temporarily 
moved to the behavioural testing room and placed in a polycarbonate holding cage. The Bluetooth-connected battery pack was attached to the test animal's intracrania device implant. The home cage was placed in the centre of a sound-attenuated box (Med-Associates). The test animal was then placed into the home cage and allowed to explore the home cage with the lid removed for $1 \mathrm{~min}$. Two minutes after this 1 min exploration, a stranger mouse (6-8 weeks) of the same sex and strain was placed into the test animal's home cage. The Bluetooth-signal-initiated $20 \mathrm{~Hz}$ stimulation period began immediately preceding the introduction of the stranger mouse. The two mice were allowed to freely interact and were video recorded via a CCD camera using Ethovision 13. Following experimentation, social interaction over the 2 min testing was manually scored and defined as any period of time in which the test mouse was actively investigating the stranger mouse. Investigation included sniffing in active contact with the stranger's snout, flank or anogenital area, grooming or pursuing the stranger as it actively explored the cage; converse investigation of the test mouse by the stranger was not scored. Each mouse underwent two social interaction tests separated in time by $1 \mathrm{~h}$, with one stranger paired with optical stimulation and one with no stimulation. Groups were counterbalanced for order of light stimulation and all behaviour was scored blind to genotype.

Scheduled activity test. Dbh-Cre $\mathrm{LC}^{\mathrm{LC}-\mathrm{mPFC}: \mathrm{ChR} 2}$ mice were connected to a fibre optic patch cable and placed into a black polycarbonate box $\left(52.5 \times 25.5 \times 25.5 \mathrm{~cm}^{3}\right)$. Animals had ad libitum access to food and water. Following $24 \mathrm{~h}$ acclimation, WNBN-initiated Bluetooth signals were scheduled (11:15 and 15:15) to be sent to a Bluetooth-connected laser to drive $473 \mathrm{~nm}$ photostimulation at $5 \mathrm{~Hz}$. Fifteen minutes before the scheduled stimulation, locomotor activity was monitored and measured for 45 min via a CCD camera using Ethovision 13

Home-cage feeding test. At 9 weeks age, standard food was removed and replaced with three specialized pelleted diets (Teklad Diets) available ad libitum and separated by metal dividers. The location of each diet was rotated daily. Behavioural testing occurred during the first hour of the dark cycle in the colony rooms. Baseline feeding procedure: the remaining home-cage specialized diets were weighed and replaced with $40 \mathrm{~g}$ pre-weighed food in a clean cage lid at the start of the dark cycle (11:00). Again, the food was weighed after $2 \mathrm{~h}$ of the dark cycle (13:00) to establish baseline intake of each diet during the first $2 \mathrm{~h}$ of the dark cycle for 1 week. Drug injection feeding procedure: at 10 weeks age, animals were subjected to the drug injection feeding procedure. Two hours before the start of the dark cycle (9:00), battery packs were placed inside the housing unit and $0.5 \mu$ l of the drug (DAMGO or saline) was loaded into the cartridge unit and placed on the device anchored to the skull. Before 11:00, the remaining home-cage specialized diets were weighed and replaced with 40 grams pre-weighed food in a clean cage lid. At lights off (11:00), delivery of the drug from the cartridge into the accumbens was administered via BLE signal. After $2 \mathrm{~h}$ (13:00), the remaining diets were weighed.

Data presentation and statistical analysis. All values are given as individual datapoints and mean \pm s.e.m., unless otherwise noted. Animal behaviour data were analysed for statistical significance by either repeated measures one-way analysis of variance (ANOVA) with Tukey's multiple comparisons correction or paired, two-tailed $t$-test as implemented in GraphPad Prism $9 .{ }^{\star} P<0.05,{ }^{\star *} P<0.01$, ${ }_{* * *}^{*}<0.001$ and ${ }^{* * * *} P<0.0001$. Parametric tests were used when data was normally distributed. Pellet interval data for FED3 experiments were graphed and analysed using FED3 Viz, a Python GUI for graphing data from FED3 devices ${ }^{33}$.

Reporting Summary. Further information on research design is available in the Nature Research Reporting Summary linked to this article.

\section{Data availability}

The main data supporting the results in this study are available within the paper and its Supplementary Information. Source data for Fig. 3 and Extended Data Fig. 1 are provided with this paper. The data collected in the animal studies are available from the corresponding authors on reasonable request. Source data are provided with this paper.

\section{Code availability}

The firmware codes used in remote control modules to enable wireless control of the animals and miscellaneous laboratory tools are available as Supplementary Information. Code used for the analyses is available from the corresponding authors on reasonable request.

Received: 6 July 2020; Accepted: 17 September 2021; Published online: 25 November 2021

\section{References}

1. Sorge, R. E. et al. Olfactory exposure to males, including men, causes stress and related analgesia in rodents. Nat. Methods 11, 629-632 (2014).
2. Olsen, C. M. \& Winder, D. G. Operant sensation seeking engages similar neural substrates to operant drug seeking in C57 mice. Neuropsychopharmacology 34, 1685-1694 (2009).

3. File, S. E., Mabbutt, P. S. \& Hitchcott, P. K. Characterisation of the phenomenon of "one-trial tolerance" to the anxiolytic effect of chlordiazepoxide in the elevated plus-maze. Psychopharmacology 102, 98-101 (1990)

4. Eckmier, A. et al. Rats can acquire conditional fear of faint light leaking through the acrylic resin used to mount fiber optic cannulas. Learn. Mem. 23, 684-688 (2016)

5. Febinger, H. Y., George, A., Priestley, J., Toth, L. A. \& Opp, M. R. Effects of housing condition and cage change on characteristics of sleep in mice. J. Am. Assoc. Lab. Anim. Sci. 53, 9 (2014).

6. Byun, S.-H. et al. Mechanically transformative electronics, sensors, and implantable devices. Sci. Adv. 5, eaay0418 (2019).

7. Jeong, J.-W. et al. Wireless optofluidic systems for programmable in vivo pharmacology and optogenetics. Cell 162, 662-674 (2015).

8. Kim, T.-I. et al. Injectable, cellular-scale optoelectronics with applications for wireless optogenetics. Science 340, 211-216 (2013).

9. Montgomery, K. L. et al. Wirelessly powered, fully internal optogenetics for brain, spinal and peripheral circuits in mice. Nat. Methods 12, 969-974 (2015).

10. Noh, K. N. et al. Miniaturized, battery-free optofluidic systems with potential for wireless pharmacology and optogenetics. Small 14, 1702479 (2018).

11. Park, S. I. et al. Stretchable multichannel antennas in soft wireless optoelectronic implants for optogenetics. Proc. Natl Acad. Sci. USA 113, E8169-E8177 (2016).

12. Qazi, R., Kim, C. Y., Byun, S.-H. \& Jeong, J.-W. Microscale inorganic LED based wireless neural systems for chronic in vivo optogenetics. Front. Neurosci. 12, 764 (2018).

13. Shin, G. et al. Flexible near-field wireless optoelectronics as subdermal implants for broad applications in optogenetics. Neuron $\mathbf{9 3}$, 509-521.e3 (2017).

14. Kim, W. S. et al. Organ-specific, multimodal, wireless optoelectronics for high-throughput phenotyping of peripheral neural pathways. Nat. Commun. 12, 157 (2021)

15. Shao, J. et al. Smartphone-controlled optogenetically engineered cells enable semiautomatic glucose homeostasis in diabetic mice. Sci. Transl. Med. 9, eaal2298 (2017).

16. Reinert, J. K., Schaefer, A. T. \& Kuner, T. High-throughput automated olfactory phenotyping of group-housed mice. Front. Behav. Neurosci. 13, 267 (2019).

17. Parkison, S. A. et al. A low-cost, reliable, high-throughput system for rodent behavioral phenotyping in a home cage environment. Annu. Int. Conf. IEEE Eng. Med. Biol. Soc. https://doi.org/10.1109/EMBC.2012.6346445 (2012).

18. Beraldo, F. H. et al. MouseBytes, an open-access high-throughput pipeline and database for rodent touchscreen-based cognitive assessment. eLife $\mathbf{8}$, e49630 (2019)

19. Qazi, R. et al. Wireless optofluidic brain probes for chronic neuropharmacology and photostimulation. Nat. Biomed. Eng. 3, 655-669 (2019)

20. Kim, C. Y. et al. Soft subdermal implant capable of wireless battery charging and programmable controls for applications in optogenetics. Nat. Commun. 12, 535 (2021)

21. Zaruba, G. V., Basagni, S. \& Chlamtac, I. Bluetrees-scatternet formation to enable Bluetooth-based ad hoc networks. In ICC 2001. IEEE International Conference on Communications. Conference Record (Cat. No.01CH37240) (IEEE, 2001).

22. Gutruf, P. et al. Fully implantable optoelectronic systems for battery-free, multimodal operation in neuroscience research. Nat. Electron. 1, 652-660 (2018)

23. Park, S. I. et al. Soft, stretchable, fully implantable miniaturized optoelectronic systems for wireless optogenetics. Nat. Biotechnol. 33, 1280-1286 (2015).

24. McCall, J. G. et al. Preparation and implementation of optofluidic neural probes for in vivo wireless pharmacology and optogenetics. Nat. Protoc. 12, 219-237 (2017)

25 . Lee, J. et al. Rapidly customizable, scalable 3D-printed wireless optogenetic probes for versatile applications in neuroscience. Adv. Funct. Mater. 30, 2004285 (2020)

26. Aponte, Y., Atasoy, D. \& Sternson, S. M. AGRP neurons are sufficient to orchestrate feeding behavior rapidly and without training. Nat. Neurosci. 14, 351-355 (2011)

27. Atasoy, D., Betley, J. N., Su, H. H. \& Sternson, S. M. Deconstruction of a neural circuit for hunger. Nature 488, 172-177 (2012).

28. Gradinaru, V. et al. Targeting and readout strategies for fast optical neural control in vitro and in vivo. J. Neurosci. 27, 14231-14238 (2007).

29. Gunaydin, L. A. et al. Natural neural projection dynamics underlying social behavior. Cell 157, 1535-1551 (2014). 
30. Mahn, M. et al. High-efficiency optogenetic silencing with soma-targeted anion-conducting channelrhodopsins. Nat. Commun. 9, 4125 (2018).

31. Klapoetke, N. C. et al. Independent optical excitation of distinct neural populations. Nat. Methods 11, 338-346 (2014).

32. Will, M. J., Franzblau, E. B. \& Kelley, A. E. Nucleus accumbens mu-opioids regulate intake of a high-fat diet via activation of a distributed brain network. J. Neurosci. 23, 2882-2888 (2003)

33. Matikainen-Ankney, B. A. et al. An open-source device for measuring food intake and operant behavior in rodent home-cages. eLife 10, e66173 (2021)

34. Baert, M., Rossey, J., Shahid, A. \& Hoebeke, J. The Bluetooth Mesh standard: an overview and experimental evaluation. Sensors 18, 2409 (2018).

35. Carter, M. E. et al. Tuning arousal with optogenetic modulation of locus coeruleus neurons. Nat. Neurosci. 13, 1526-1533 (2010).

36. Sparta, D. R. et al. Construction of implantable optical fibers for long-term optogenetic manipulation of neural circuits. Nat. Protoc. 7, 12-23 (2012).

37. Mousel, M. R., Stroup, W. W. \& Nielsen, M. K. Locomotor activity, core body temperature, and circadian rhythms in mice selected for high or low heat loss. J. Anim. Sci. 79, 861 (2001).

38. Redfern, W. S. et al. Automated recording of home cage activity and temperature of individual rats housed in social groups: The Rodent Big Brother project. PLoS ONE 12, e0181068 (2017).

39. Wentz, C. T. et al. A wirelessly powered and controlled device for optical neural control of freely-behaving animals. J. Neural Eng. 8, 046021 (2011).

40. McCall, J. G. et al. Fabrication and application of flexible, multimodal light-emitting devices for wireless optogenetics. Nat. Protoc. 8, 2413-2428 (2013).

41. Dagnew, R. et al. CerebraLux: a low-cost, open-source, wireless probe for optogenetic stimulation. Neurophotonics 4, 045001 (2017).

42. Pais-Vieira, M., Lebedev, M., Kunicki, C., Wang, J. \& Nicolelis, M. A. L. A brain-to-brain interface for real-time sharing of sensorimotor information. Sci. Rep. 3, 1319 (2013).

43. Russell, W. M. S. \& Burch, R. L. The Principles of Humane Experimental Technique (Methuen, 1958)

44. Yin, M. et al. Wireless neurosensor for full-spectrum electrophysiology recordings during free behavior. Neuron 84, 1170-1182 (2014).

45. Shuman, T. et al. Breakdown of spatial coding and interneuron synchronization in epileptic mice. Nat. Neurosci. 23, 229-238 (2020).

46. Reinhold, A. S., Sanguinetti-Scheck, J. I., Hartmann, K. \& Brecht, M. Behavioral and neural correlates of hide-and-seek in rats. Science 365 1180-1183 (2019)

47. Crawford, L. E. Enriched environment exposure accelerates rodent driving skills. Behav. Brain Res. 378, 112309 (2020).

48. Servick, K. et al. Updated: labs go quiet as researchers brace for long-term coronavirus disruptions. ScienceInsider https://www.sciencemag.org/news/ 2020/03/updated-labs-go-quiet-researchers-brace-long-term-coronavirusdisruptions (2020).

49. Paxinos, G., Watson, C. R. R. \& Emson, P. C. AChE-stained horizontal sections of the rat brain in stereotaxic coordinates. J. Neurosci. Methods 3 , 129-149 (1980).
50. Faul, F., Erdfelder, E., Lang, A.-G. \& Buchner, A. G*Power 3: a flexible statistical power analysis program for the social, behavioral, and biomedical sciences. Behav. Res. Methods 39, 175-191 (2007).

51. Krashes, M. J. et al. Rapid, reversible activation of AgRP neurons drives feeding behavior in mice. J. Clin. Invest. 121, 1424-1428 (2011).

52. Krashes, M. J. et al. An excitatory paraventricular nucleus to AgRP neuron circuit that drives hunger. Nature 507, 238-242 (2014).

\section{Acknowledgements}

This work was supported by the KAIST-funded Global Singularity Research Program (J.-W.J.), the Basic Science Research Program through the National Research Foundation of Korea (NRF) funded by the Ministry of Science and ICT (NRF-2021R1A2C4001483 and NRF-2020M3A9G8018572, J.-W.J.), the National Institutes of Health (R01NS117899, J.G.M.) and the Oak Ridge Associated Universities (J.G.M.). This work was also supported by the United States National Institutes of Health (R25 MH112473, J.B.) and the Hope Center Viral Vectors Core.

\section{Author contributions}

R.Q., K.E.P., J.G.M. and J.-W.J. conceived the project and designed the detailed experimental protocols. R.Q. and C.Y.K. designed and fabricated the neural probes and wireless RCM hardware for behavioural tests. R.Q. and C.Y.K. designed the firmware for the wireless RCM hardware. K.E.P., M.R.N., J.B., J.R.K., M.C.W. and G.B.G. performed the mouse experiments. G.B.G., J.R.L., M.A.T., M.J.W. and J.G.M. performed the rat experiments. R.Q., C.Y.K., R.R., J.C., H.L., Y.X., S.H. and J.-W. J. conceptualized and designed the software for local piconet control and global Internet control. R.Q., K.E.P., C.Y.K., R.R., A.V.K., S.H., J.G.M. and J.-W.J. performed the investigation and analysed the data. R.Q., K.E.P., C.Y.K., J.C., S.H., J.G.M. and J.-W.J. wrote the paper. S.H., J.G.M. and J.-W.J. acquired funding and supervised the project. S.H., J.G.M. and J.-W.J. are co-senior authors. All authors discussed the results and contributed to the revision of the manuscript.

\section{Competing interests}

The authors declare no competing interests.

\section{Additional information}

Extended data is available for this paper at https://doi.org/10.1038/s41551-021-00814-w. Supplementary information The online version contains supplementary material available at https://doi.org/10.1038/s41551-021-00814-w.

Correspondence and requests for materials should be addressed to Sangtae Ha, Jordan G. McCall or Jae-Woong Jeong.

Peer review information Nature Biomedical Engineering thanks Jit Muthuswamy, Bozhi Tian and Chong Xie for their contribution to the peer review of this work. Peer reviewer reports are available.

Reprints and permissions information is available at www.nature.com/reprints.

Publisher's note Springer Nature remains neutral with regard to jurisdictional claims in published maps and institutional affiliations.

(c) The Author(s), under exclusive licence to Springer Nature Limited 2021 


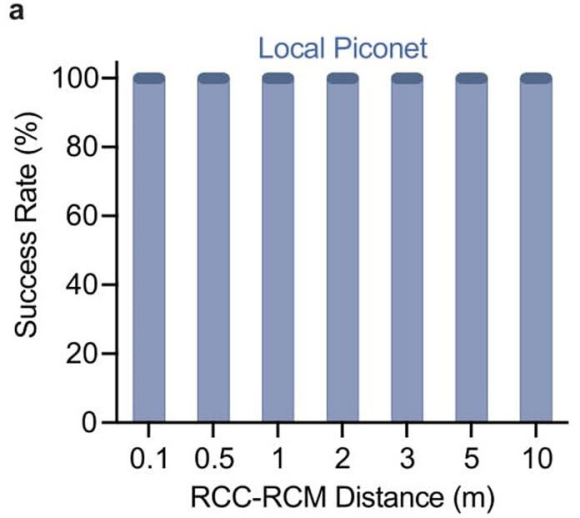

b

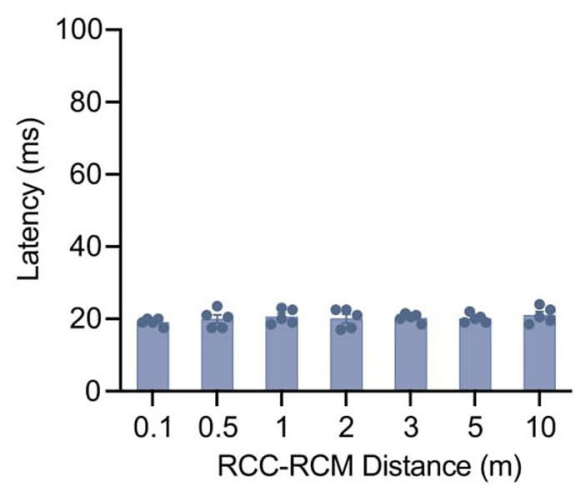

C

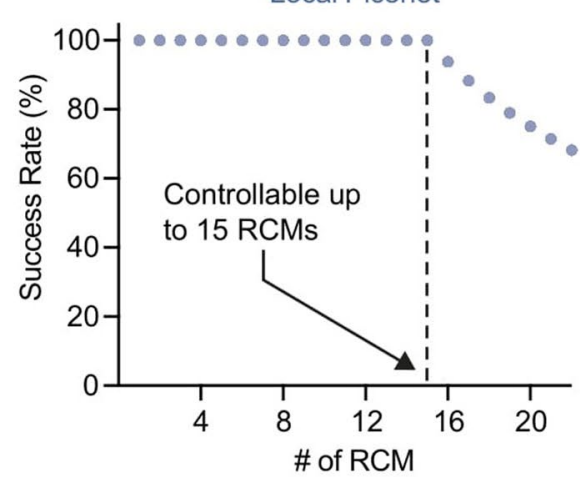

d

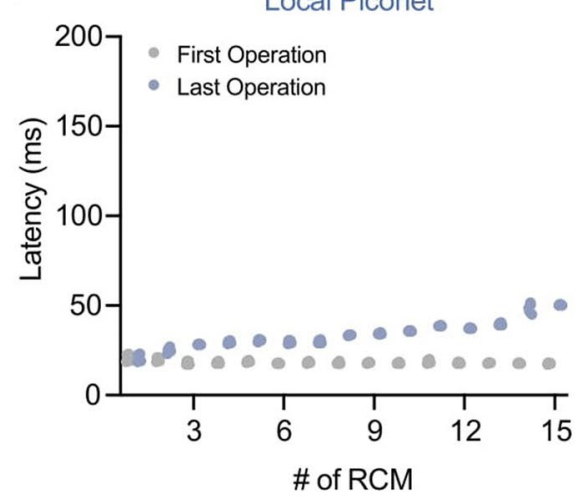

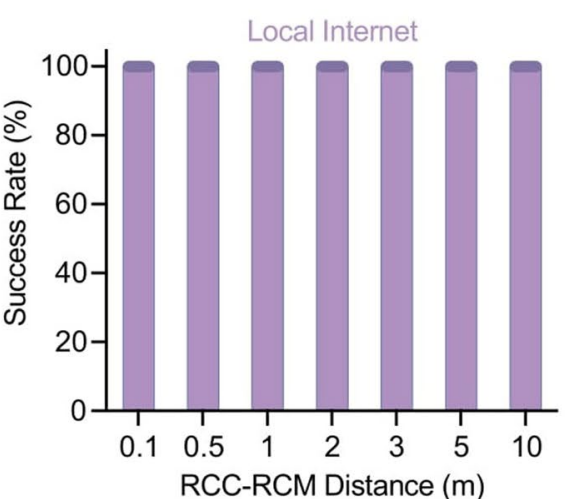

Local Internet

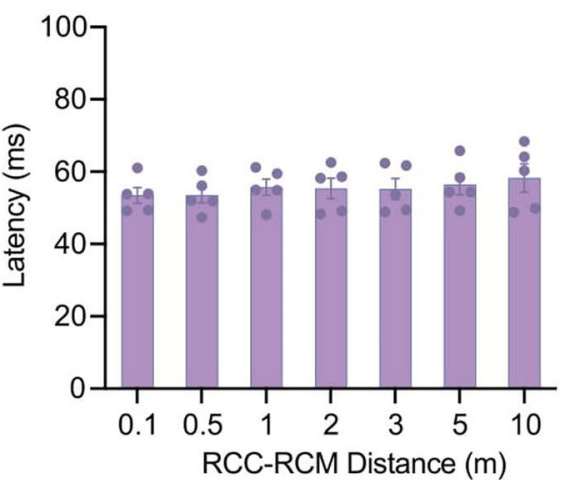

Local Internet

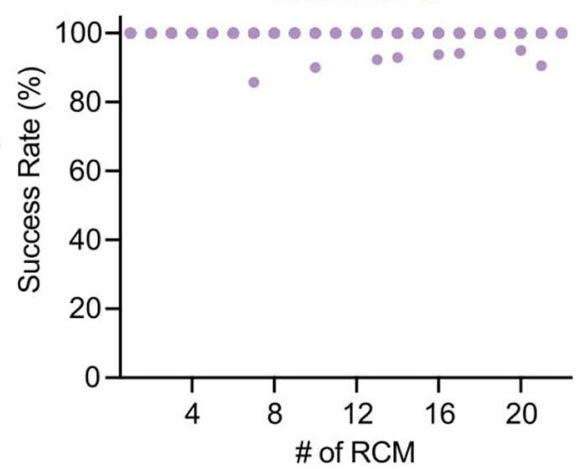

Local Internet

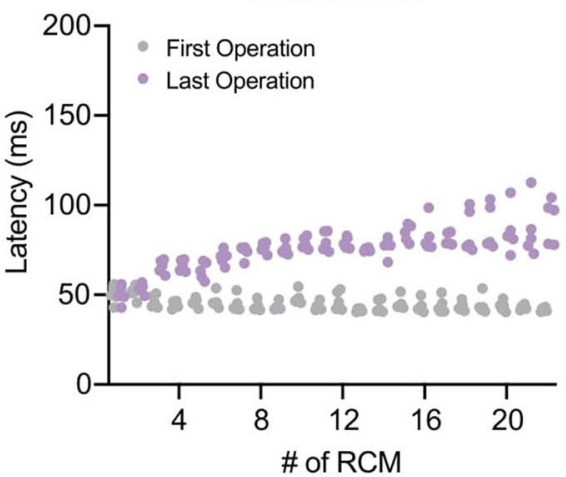

Global Internet

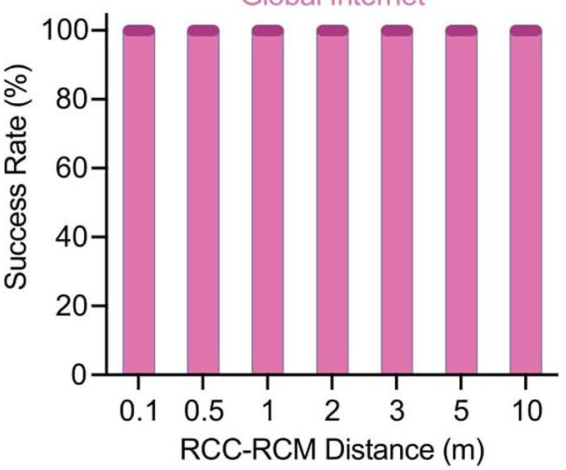

Global Internet

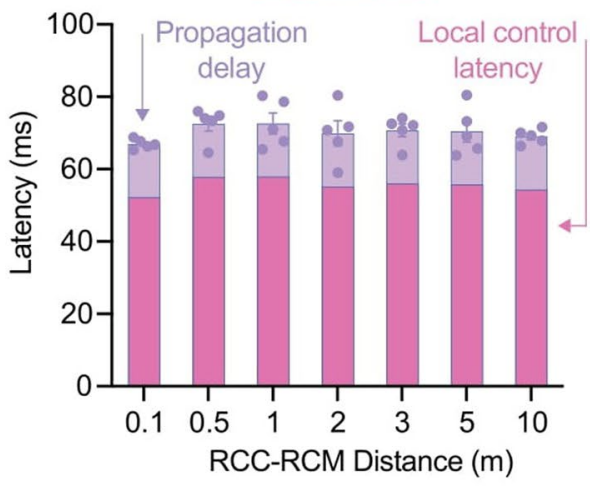

Global Internet

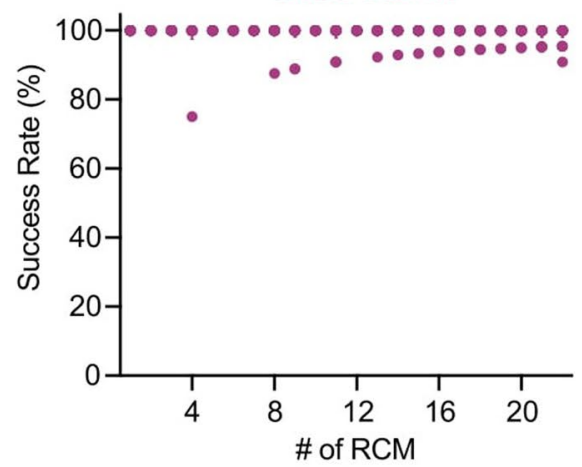

Global Internet

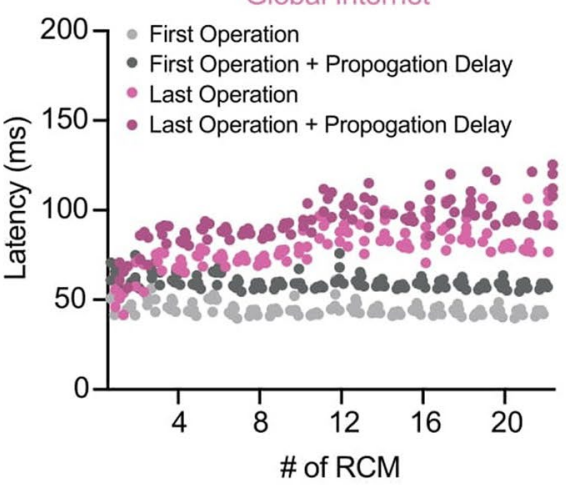

Extended Data Fig. 1 | See next page for caption. 
Extended Data Fig. 1 | Performance characteristics of the local and global WNBN. a-b, Success rate $(\mathbf{a} ; n=20)$ and latency $(\mathbf{b} ; n=5)$ of triggering $\mu$-ILED neural probes with wireless RCMs as a function of different RCC-RCM distances $(0.1,0.5,1,2,3,5$, and $10 \mathrm{~m})$, comparing the responsiveness of local piconet, local internet, and global internet modes. The purple-color parts in the global internet latency graph (b, right) indicates a signal propagation delay, which is dependent on the inter-distance between the remote user and RCC (for example, 15 ms delay between Boulder, CO and St. Louis, MO, USA). $\mathbf{c}-\mathbf{d}$, Success rate $(\mathbf{c} ; n=20)$ and latency $(\mathbf{d} ; n=5)$ of triggering a $\mu$-ILED in optogenetic neural probe integrated with wireless RCMs as a function of different numbers of RCMs (1-22; $1 \mathrm{~m}$ distance from RCC), comparing the responsiveness of local piconet, local internet, and global internet modes. Note that the latency of the global internet control is determined by summing the local control latency and the distance-dependent propagation delay. 
a

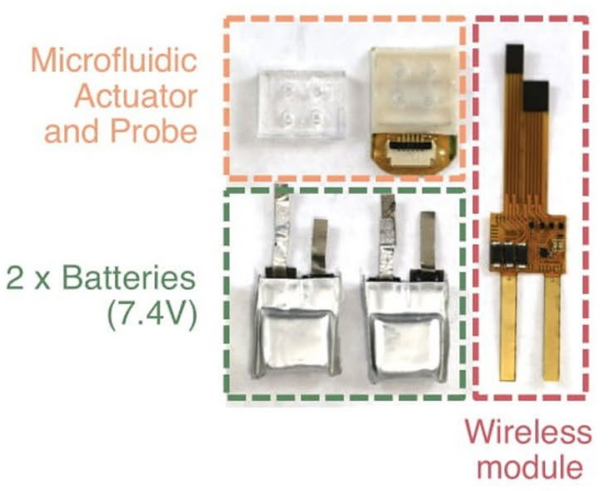

b

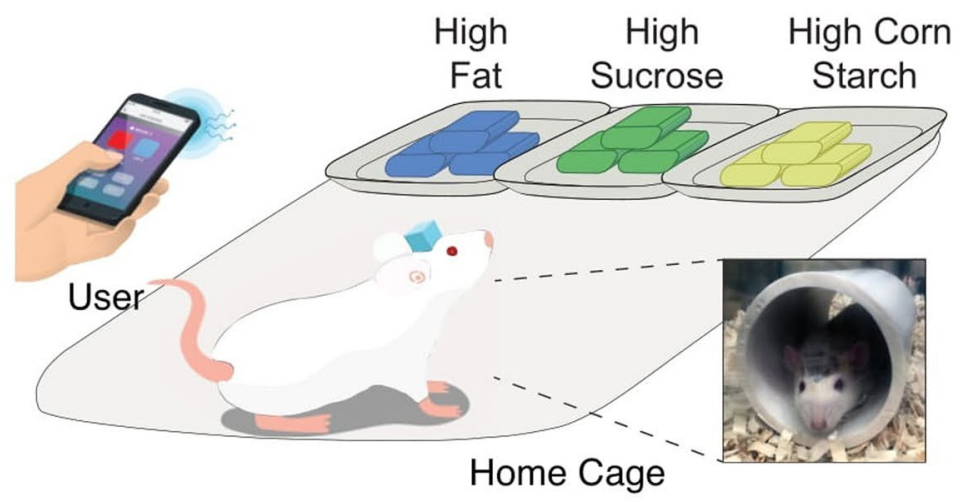

e

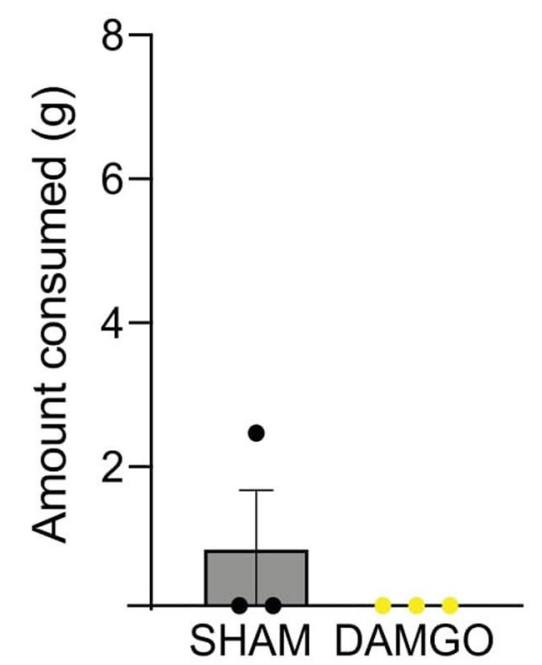

SHAM DAMGO

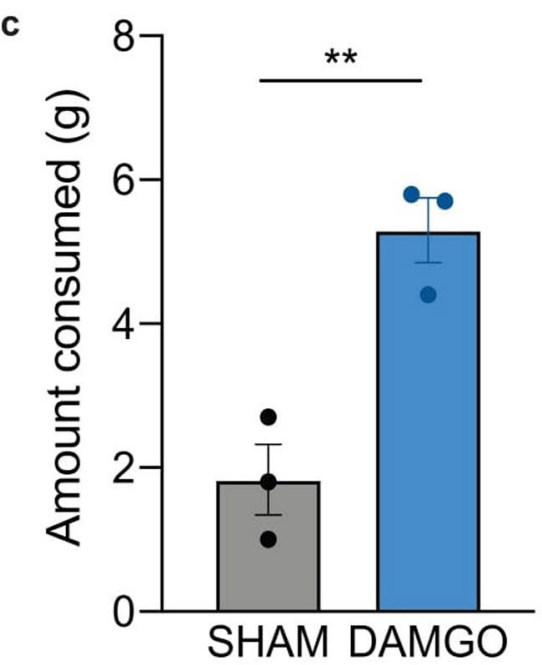

d

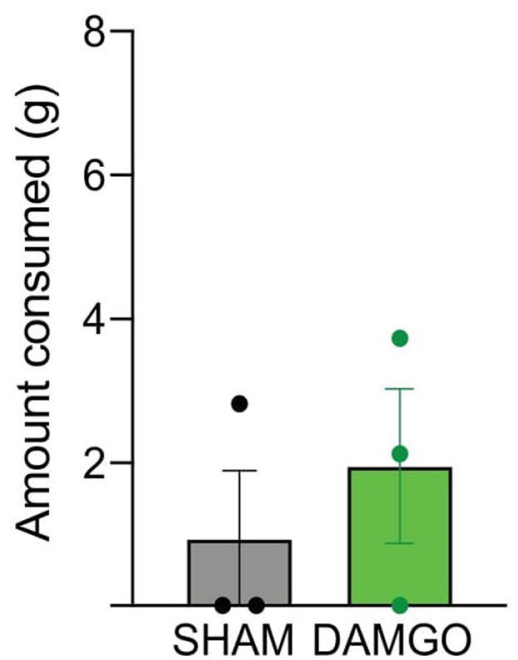

Extended Data Fig. 2 | Local Piconet control of microfluidic drug delivery in rats. a, Representative photographs of wireless microfluidic device components. b. Cartoon schematic of behavioural setup evaluating ad libitum food consumption following locally controlled microfluidic administration of $\mu$-opioid agonist, DAMGO. Inset, photograph of rat with implanted microfluidic device. c-e, Nucleus accumbens DAMGO delivery increases binge feeding of the high fat (c), but not high sucrose (d) or high corn starch (e) diets (Two tailed paired t-test, ${ }^{\star \star} P=0.0045, n=3$ ). 


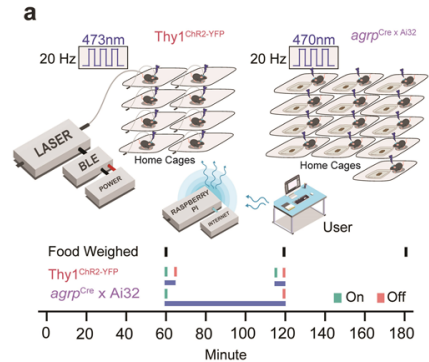

d

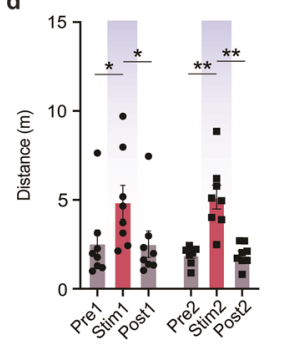

e

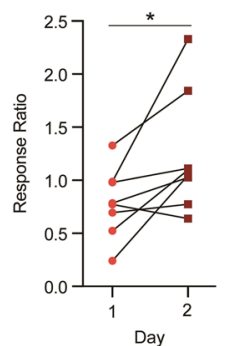

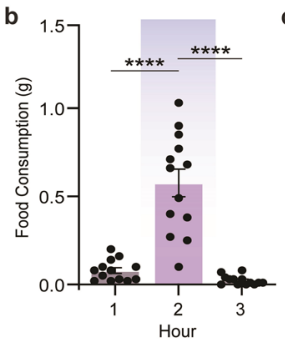

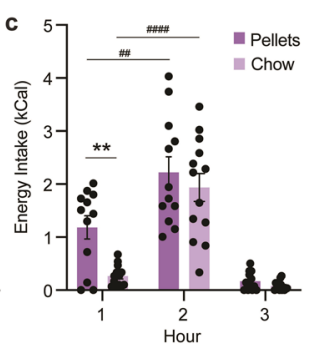

f

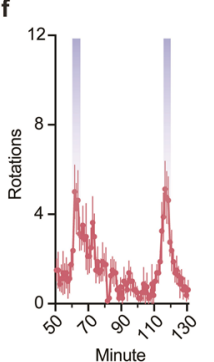

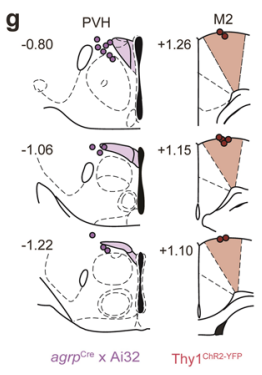

Extended Data Fig. 3 | Simultaneous Internet control of high throughput behavioural experimentation. a, Cartoons and timelines describing the locomotion and ad libitum home cage chow feeding experiment with internet-scheduled simultaneous control. $\mathbf{b}$, Average amount of home cage chow consumed during 1 hour epochs, purple bar depicts hour of during $20 \mathrm{~Hz}$ stimulation. agrp ${ }^{\mathrm{Cre}} \times \mathrm{Ai} 32$ mice consume substantially more food during $20 \mathrm{~Hz}$ photostimulation compared to non-stimulation epochs (1 hour) (repeated measures one-way ANOVA, Hour 1 vs Hour 2, ${ }^{\star \star \star \star} p<0.0001$, Hour 2 vs Hour 3 , ${ }^{\star \star \star \star} P<0.0001$, Tukey's multiple comparisons test, $n=13$ ). c, Average caloric intake of home cage chow and pellets during 1 hour epochs, purple bar depicts hour of during $20 \mathrm{~Hz}$ stimulation. agrp ${ }^{\mathrm{Cre}} \times \mathrm{Ai} 32$ mice have substantially higher caloric during $20 \mathrm{~Hz}$ photostimulation compared to non-stimulation

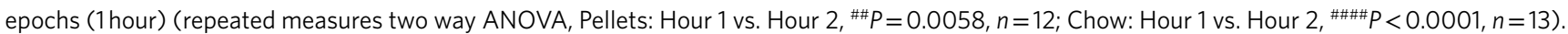
agrp $^{\text {Cre }} \times$ Ai32 mice also have substantially higher caloric intake of the pellets as compared to the home cage show during the initial presentation of food (Hour 1) (repeated measures two-way ANOVA, Hour 1: Pellets vs. Chow, ${ }^{\star \star} P=0.0231, n=12,13$ ). d, Data showing changes in locomotor activity during internet-controlled stimulation $(20 \mathrm{~Hz})$ in Thy $7^{\text {ChR2-YFP }}$ mice. Average distance travelled during internet-scheduled stimulation is substantially higher than pre-stimulation and post-stimulation epochs (repeated measures one-way ANOVA, for Stimulation 1: Pre1 vs Stim1, ${ }^{\star} P=0.0292$ and Stim1 vs Post1 ${ }^{\star} P=0.0147, n=8$, Tukey's multiple comparisons test; For Stimulation 2: Pre2 vs Stim2 ${ }^{\star \star} P=0.0014$ and Stim2 vs Post2 ${ }^{\star \star} P=0.0048, n=8$ ). e, Relative response ratio between the first and second Thy $7^{\text {ChR2-YFP }}$ stimulations on each day indicates that the second stimulation produced a more robust locomotor response on the second experimental day (Two tailed paired t-test, ${ }^{\star} P=0.0325, n=8$ ). $\mathbf{f}$, Rotations in Thy ${ }^{\text {ChR2-rFP }}$ mice during one minute bins over 80 minutes. $\mathbf{g}$, Coronal brain atlas diagrams showing targeting of $\mu$-ILED devices to the PVH of agrp ${ }^{\text {Cre }} \times$ Ai32 mice (left panel) and fiber optic implants to the $\mathrm{M} 2$ of Thy $^{1 \text { ChR2 }}$ mice. 


\section{Reporting Summary}

Nature Portfolio wishes to improve the reproducibility of the work that we publish. This form provides structure for consistency and transparency in reporting. For further information on Nature Portfolio policies, see our Editorial Policies and the Editorial Policy Checklist.

\section{Statistics}

For all statistical analyses, confirm that the following items are present in the figure legend, table legend, main text, or Methods section.

n/a Confirmed

$\bigotimes$ The exact sample size $(n)$ for each experimental group/condition, given as a discrete number and unit of measurement

$\bigotimes$ A statement on whether measurements were taken from distinct samples or whether the same sample was measured repeatedly

The statistical test(s) used AND whether they are one- or two-sided

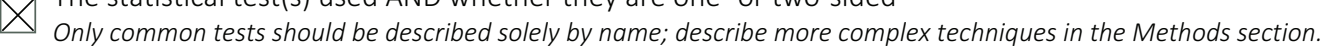

\ $\square$ A description of all covariates tested

$\bigotimes$ A description of any assumptions or corrections, such as tests of normality and adjustment for multiple comparisons

A full description of the statistical parameters including central tendency (e.g. means) or other basic estimates (e.g. regression coefficient)

$\triangle$ AND variation (e.g. standard deviation) or associated estimates of uncertainty (e.g. confidence intervals)

For null hypothesis testing, the test statistic (e.g. $F, t, r$ ) with confidence intervals, effect sizes, degrees of freedom and $P$ value noted

Give P values as exact values whenever suitable.

Х $\square$ For Bayesian analysis, information on the choice of priors and Markov chain Monte Carlo settings

Х $\square$ For hierarchical and complex designs, identification of the appropriate level for tests and full reporting of outcomes

Х $\square$ Estimates of effect sizes (e.g. Cohen's $d$, Pearson's $r$ ), indicating how they were calculated

Our web collection on statistics for biologists contains articles on many of the points above.

\section{Software and code}

Policy information about availability of computer code

Data collection Ethovision 13 (Noldus Information Technologies, Leesburg, VA) was used to record and quantify locomotion and exploration behaviours.

Data analysis Ethovision 13, GraphPad Prism 9, and FED3 Viz (Python).

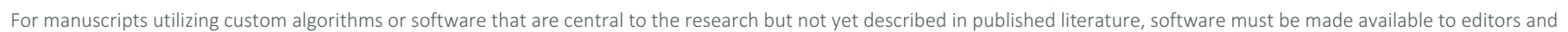

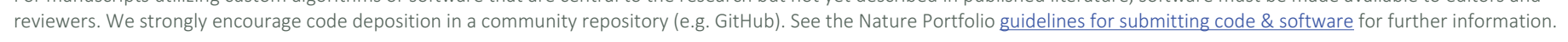

\section{Data}

Policy information about availability of data

All manuscripts must include a data availability statement. This statement should provide the following information, where applicable:

- Accession codes, unique identifiers, or web links for publicly available datasets

- A description of any restrictions on data availability

- For clinical datasets or third party data, please ensure that the statement adheres to our policy

The authors declare that the main data supporting the results in this study are available within the paper and its Supplementary Information. Source data for Fig. 3 and Extended Fig. 1 are provided with this paper. The data collected in the animal studies is available from the corresponding authors on reasonable request. 


\section{Field-specific reporting}

Please select the one below that is the best fit for your research. If you are not sure, read the appropriate sections before making your selection.

\Life sciences

Behavioural \& social sciences

Ecological, evolutionary \& environmental sciences

For a reference copy of the document with all sections, see nature.com/documents/nr-reporting-summary-flat.pdf

\section{Life sciences study design}

All studies must disclose on these points even when the disclosure is negative.

Sample size The starting sample sizes selected were determined with $\mathrm{G}^{*}$ Power3, on the basis of a power of 0.85 , or estimated from published data (or, in some cases, from small pilot studies run for this purpose).

Data exclusions Animals were excluded if any of these three conditions applied: 1) viral expression was not achieved at the target site; 2 ) the fibre-optic, $\mu$ ILED device or microfluidic device were not in the targeted brain regions; or 3) there was indication from video or WNBN logs that stimulation did not occur.

Replication All attempts at replication were successful.

Randomization All animals were randomly assigned to test groups within a given treatment group.

Blinding Investigators were blinded to each group during data collection and analysis. An exception was within-subject tests, where the experimenter knew that all animals received all treatments. In all cases, the experimenter was blind to the video analyses.

\section{Reporting for specific materials, systems and methods}

We require information from authors about some types of materials, experimental systems and methods used in many studies. Here, indicate whether each material, system or method listed is relevant to your study. If you are not sure if a list item applies to your research, read the appropriate section before selecting a response.

Materials \& experimental systems

n/a Involved in the study

$\square \bigotimes$ Antibodies

\ $\square$ Eukaryotic cell lines

Methods

\ $\square$ Palaeontology and archaeology

$\mathrm{n} / \mathrm{a}$ Involved in the study

X $\square$ chip-seq

X $\square$ Flow cytometry

$\square$ Animals and other organisms

Х MRI-based neuroimaging

Х $\square$ Human research participants

Х $\square$ Clinical data

\ $\square$ Dual use research of concern

\section{Antibodies}

Antibodies used

Validation
Chicken Anti-Tyrosine hydroxylase, Aves Labs, Cat\#TH, AB_10013440

Rabbit anti-ChR2, American Research Products, Cat\# 03-651180

From the supplier: Antibodies were analyzed by immunohistochemistry (at a concentration of $3 \mu \mathrm{g} / \mathrm{mL}$ ) using fluorescein-labelled goat anti-chicken IgY (1:500 dilution, Aves Labs Cat.\# F-1005) as the secondary reagent.

We also validated this antibody in the following published papers: McCall, J. G. et al. CRH Engagement of the Locus Coeruleus Noradrenergic System Mediates Stress-Induced Anxiety. Neuron (2015) doi:10.1016/j.neuron.2015.07.002. Kim, T. et al. Injectable, cellular-scale optoelectronics with applications for wireless optogenetics. Science 340, 211-216 (2013).

\section{Animals and other organisms}

Policy information about studies involving animals; ARRIVE guidelines recommended for reporting animal research

Laboratory animals

Wild animals

Field-collected samples
Mice: C57BL/6J, DAT-IRES-Cre, Thy1-ChR2-YFP, Dbh-IRES-Cre, and AgRP-IRES-Cre crossed with Ai32 mice. Male and female mice, aged 3-6 months, were used for each experiment.

Male Wistar rats, 2-months old.

The study did not involve wild animals.

The study did not involve samples collected from the field. 
